\title{
A Moisture-Stratiform Instability for Convectively Coupled Waves
}

\section{Citation}

Kuang, Zhiming M. 2008. A moisture-stratiform instability for convectively coupled waves. Journal of Atmospheric Sciences 65, no. 3: 834-854.

\section{Published Version}

http://dx.doi.org/10.1175/2007JAS2444.1

\section{Permanent link}

http://nrs.harvard.edu/urn-3:HUL.InstRepos:3203007

\section{Terms of Use}

This article was downloaded from Harvard University's DASH repository, and is made available under the terms and conditions applicable to Other Posted Material, as set forth at http:// nrs.harvard.edu/urn-3:HUL.InstRepos:dash.current.terms-of-use\#LAA

\section{Share Your Story}

The Harvard community has made this article openly available.

Please share how this access benefits you. Submit a story.

\section{Accessibility}




\section{A moisture-stratiform instability for convectively coupled}

\section{waves}

Zhiming Kuang

Department of Earth and Planetary Sciences and School of Engineering and Applied

Sciences, Harvard University

Submitted to the Journal of the Atmospheric Sciences, March 2, 2007

Revised, June 14, 2007

Corresponding author address:

Zhiming Kuang

Department of Earth and Planetary Sciences and School of Engineering and Applied Sciences, Harvard University, 20 Oxford St. Cambridge, MA 02138

(Tel) 617 495-2354

(Fax) 617-495-7660

Email:kuang@fas.harvard.edu 


\begin{abstract}
A simple model of two vertical modes is constructed and analyzed to reveal the basic instability mechanisms of convectively coupled waves. The main novelty of this model is a convective parameterization based on the quasi-equilibrium concept and simplified for a model of two vertical modes. It hypothesizes 1) approximate invariance of the difference between saturation moist static energy in the lower half of the troposphere and moist static energy in the sub-cloud layer, regardless of free troposphere humidity, 2) variations in the depth of convection is determined by moisture deficit variations in the mid-troposphere. Physical arguments for such a treatment are presented. For realistic model parameters chosen based on cloud system resolving model simulations (CSRM) of an earlier study, the model produces unstable waves at wavelengths and with structures that compare well with the CSRM simulations and observations.
\end{abstract}

A moisture-stratiform instability and a direct stratiform instability are identified as the main instability mechanisms in the model. The former relies on the effect of midtroposphere humidity on the depth of convection. The latter relies on the climatological mean convective heating profile being top heavy, and is identified to be the same as the stratiform instability mechanism proposed by Mapes (2000). The moisture-stratiform instability appears to be the main instability mechanism for the convectively couple wave development in the CSRM simulations. The finite response time of convection has a damping effect on the waves that is stronger at high wavenumbers. The net moistening effect of the second mode convective heating also damps the waves, but more strongly at low wavenumbers. These effects help to shape the growth rate curve so that the most unstable waves are of a few thousand kilometers in scale. 


\section{Introduction}

There is a long history of constructing models with gross vertical structures to capture the basic dynamics of convectively coupled tropical waves (e.g. Lindzen, 1974;Emanuel, 1987;Neelin et al., 1987;Wang, 1988;Mapes, 2000;Majda and Shefter, 2001;Khouider and Majda, 2006). Earlier models emphasized a first baroclinic structure (or mode) that is of one sign over the full depth of the free troposphere (e.g. Lindzen, 1974;Emanuel, 1987; Neelin et al., 1987;Wang, 1988). More recent observations of these waves revealed a significant second baroclinic component in their vertical temperature structures (e.g. Wheeler et al., 2000;Straub and Kiladis, 2002;Haertel and Kiladis, 2004). Such observations alone do not contradict models based only on the first baroclinic mode; it is possible that the first baroclinic mode captures the basic dynamics and the second mode temperature structure is simply a byproduct. The more convincing evidence for the inadequacy of the first baroclinic mode models is that they do not yield instability without external destabilization mechanisms (Emanuel et al., 1994), which is inconsistent with the results of cloud system resolving model (CSRM) simulations (e.g. Tulich et al., 2006;Kuang, 2007). Mapes (2000) proposed the first instability model that contains both the first and the second baroclinic modes, and identified a stratiform instability for the wave-convection coupling (Mapes, 2000), hereafter M00. The behavior of such models has been analyzed in some detail (Mapes, 2000;Majda and Shefter, 2001;Majda et al., 2004). In these models, modulation of convection by the second mode temperature anomaly is emphasized, and effects of free troposphere moisture variations are ignored. Results from Kuang (2007), hereafter K07, however, indicate that moisture is an essential component for allowing convectively coupled waves to develop. Moisture was included 
in the two vertical mode model of Khouider and Majda, (2006a), hereafter KM06, along with a third cloud type, congestus (in addition to the deep convective and stratiform clouds in M00). While considerable analyses have been done on the linear stability of this model (KM06; Khouider and Majda, 2006b), its convective parameterization is rather complicated and an instability mechanism involving moisture was not clearly identified.

This study continues the effort to construct models of convectively coupled waves with crude vertical structures. Our emphasis will be on conceptually simple convective parameterizations and on revealing the model's basic instability mechanisms. The model formulation is presented in section 2, along with results from linear analyses. The model parameters are selected based on CSRM simulations of convectively coupled waves described in $\mathrm{K} 07$ so that the model resides in realistic parameter regimes (Appendix A). The model is then simplified further and limiting cases are considered to reveal its basic instability mechanisms (section 3). This is followed by a summary and discussion section (section 4) and three appendices.

\section{Formulation and linear analyses of the simple model}

Like earlier models (e.g. M00; Majda and Shefter, 2001, hereafter MS01; KM06), the present model has two components: the first describes the response to convective heating and the second describes the convective parameterization.

\section{a. Response to convective heating}

Similar to K07, we start with the linear inviscid anelastic 2D primitive equations for a horizontal wavenumber $\mathrm{k}$ with a background state of no motion, and eliminate pressure and horizontal winds. This gives 


$$
\begin{aligned}
& \left(\frac{\partial}{\partial t}+\varepsilon\right)\left(\bar{\rho} w^{\prime}\right)_{z z}=-k^{2} \bar{\rho} g \frac{T^{\prime}}{\bar{T}} \\
& \frac{\partial}{\partial t} T^{\prime}+w^{\prime}\left(\frac{d \bar{T}}{d z}+\frac{g}{c_{p}}\right)=J^{\prime}
\end{aligned}
$$

where $\varepsilon$ is the mechanical damping coefficient, taken to be a constant, $\mathrm{J}$ is convective heating, and all other symbols assume their usual meteorological meaning. The overbar denotes the background mean variables and prime denotes deviations from the mean. Despite the various assumptions and simplifications, systems such as Eq. (1) capture well the basic wind and temperature distributions of convectively coupled waves given the convective heating and cooling. This was shown for the 2-day waves (Haertel and Kiladis, 2004) and is true for the present case as well (not shown).

We then assume rigid plate boundary conditions at the surface and at the top of the troposphere and expand the forcing and the solution in terms of the vertical eigenmodes $\left(\mathrm{G}_{\mathrm{i}}\right)$ :

$$
\begin{aligned}
& \bar{\rho} w^{\prime}\left(\frac{d \bar{T}}{d z}+\frac{g}{c_{p}}\right)=\sum_{j=1}^{2} w_{j}(x, t) G_{j}(z)\left(\frac{d \bar{T}}{d z}+\frac{g}{c_{p}}\right) \\
& \bar{\rho} T^{\prime}=\sum_{j=1}^{2} T_{j}(x, t) G_{j}(z)\left(\frac{d \bar{T}}{d z}+\frac{g}{c_{p}}\right) \\
& \bar{\rho} J^{\prime}=\sum_{j=1}^{2} J_{j}(x, t) G_{j}(z)\left(\frac{d \bar{T}}{d z}+\frac{g}{c_{p}}\right)
\end{aligned}
$$

and obtain:

$$
\begin{aligned}
& \left(\frac{\partial}{\partial t}+\varepsilon\right) w_{j}-k^{2} c_{j}^{2} T_{j}=0 \\
& \frac{\partial}{\partial t} T_{j}+w_{j}=J_{j}
\end{aligned}
$$


Without forcing and damping, the solutions to Eq. (3) for each vertical mode are two neutral waves propagating in opposite directions with a dry wave speed of $c_{j}$. When the buoyancy frequency, the square of which is $N^{2}=g\left(\frac{d \ln \bar{T}}{d z}+\frac{g}{c_{p} \bar{T}}\right)$, is constant, the vertical modes are

$$
G_{j}(z)=\frac{\pi}{2} \sin \left(\frac{j \pi z}{H_{T}}\right)
$$

where $\mathrm{H}_{\mathrm{T}}$ is the height of the troposphere. The modes are normalized so that their absolute values average to unity over the depth of the troposphere. We have retained only the first two vertical modes with the goal of constructing a minimal model to reveal the basic instability mechanisms. Note that congestus and stratiform heating are treated here as opposite phases of the same mode $\left(\mathrm{J}_{2}\right)$. This simpler treatment represents the observed and CSRM simulated heating structures very well (Haertel and Kiladis, 2004; K07).

It is useful to remind ourselves the empirical nature of the two-mode model: the two vertical modes are chosen not because they are mathematically the first two eigenmodes of Eq. 1 with the rigid plate boundary conditions. They are chosen because of the empirical evidence that basic vertical structure of the waves can be captured with these two modes (e.g. Haertel and Kiladis, 2004; K07). Indeed, it is based on this empirical evidence and the evidence that a radiation upper boundary condition does not have a major effect on the wave characteristics (K07) that the rigid plate boundary conditions were then chosen, allowing Eq. 1 to be conveniently decomposed into vertical modes that resemble the vertical modes seen empirically. Therefore, the present model does not 
address why these particular vertical structures/modes dominate, the answer to which requires a model that allows vertical modes to be selected naturally.

Eq (3) is augmented by an equation for the subcloud layer moist static energy $h_{b}$ and an equation for the mid-tropospheric humidity $\mathrm{q}_{\text {mid. }}$. The equation for $\mathrm{h}_{\mathrm{b}}$ follows prior studies (e.g. M00, MS01, KM06):

$$
\frac{\partial h_{b}}{\partial t}=E-b_{1} J_{1}-b_{2} J_{2}
$$

where $E$ is the tendency from surface heat flux anomalies, and $b_{1}$ and $b_{2}$ represent the reduction of $h_{b}$ per unit $J_{1}$ and $J_{2}$, respectively. In reality, large-scale vertical advection has a smaller but non-negligible effect on $\mathrm{h}_{\mathrm{b}}$, although including them does not appear to change the basic behaviors of the model so they are left out for simplicity. In this paper, we further set $E=0$ to eliminate any surface heat flux feedbacks, which, as shown in K07, do not change the basic characteristics of the waves.

The equation for $\mathrm{q}_{\mathrm{mid}}$ can be written as:

$$
\frac{\partial q_{m i d}}{\partial t}=a_{1} w_{1}+a_{2} w_{2}-d_{1} J_{1}-d_{2} J_{2}
$$

where $a_{1}$ and $a_{2}$ represent the effective moisture stratification for the two modes, $d_{1}$ and $d_{2}$ represent the convective drying effect on $q_{\text {mid }}$ per unit $J_{1}$ and $J_{2}$. We have neglected horizontal advection of moisture. The parameters $\mathrm{a}_{1}$ and $\mathrm{a}_{2}$ can be derived given the background moisture stratification and the vertical structure of $\mathrm{w}_{1}$ and $\mathrm{w}_{2}$. In KM06, vertically averaged free troposphere humidity $<\mathrm{q}>$ was used in Eq. (6) so that column moist static energy conservation can be used to constrain $d_{1}$ and $d_{2}$. However, the main purpose of including an equation for moisture is to include the role of tropospheric 
humidity on convection, as discussed in more detail in section $2 \mathrm{~b}$. While conveniently constrained by column moist static energy conservation, $<\mathrm{q}>$ is not necessarily the most relevant quantity for this purpose, even though it could serve as a reasonable approximation. In this paper, we will use the mid-tropospheric humidity $\mathrm{q}_{\text {mid }}$ instead, and forgo the convenience of using $<\mathrm{q}>$.

As discussed further in Appendix A and noted in many previous studies (e.g. Haertel and Kiladis, 2004), there is substantial compensation between adiabatic cooling and convective heating associated with the first mode, i.e. $\mathrm{w}_{1} \sim \mathrm{J}_{1}$. Furthermore, because $\mathrm{q}_{\text {mid }}$ is located around the nodal point of $\mathrm{w}_{2}$, the effect of large-scale advection by $\mathrm{w}_{2}$ on $\mathrm{q}_{\mathrm{mid}}$ (i.e. $a_{2}$ ) is small. We may therefore simplify Eq. (6) to

$$
\frac{\partial q_{m i d}}{\partial t}=m_{1} J_{1}+m_{2} J_{2}
$$

where $m_{1} \approx a_{1}-d_{1}$ and $m_{2} \approx-d_{2}$ are the moistening effects per unit $J_{1}$ and $J_{2}$. We have again verified that this simplification does not modify the basic behaviors of the model discussed in this paper.

Equations (3), (5), and (7) describe the atmosphere's response to convective heating. We non-dimensionalize the above equations using the first dry baroclinic gravity wave speed $(50 \mathrm{~m} / \mathrm{s})$ as the velocity scale so that $\mathrm{c}_{1}$ is 1 , and $\mathrm{c}_{2}$ is set to $1 / 2$. We use 1 day as the time scale, so the length scale is $4320 \mathrm{~km}$, and $T_{j}, h_{b}$, and $q$ are expressed in temperature unit ${ }^{1}$ $(1 \mathrm{~K})$ so that the scale for $\mathrm{J}_{\mathrm{j}}$ and $\mathrm{w}_{\mathrm{j}}$ is $1 \mathrm{~K} /$ day.

\footnotetext{
${ }^{1} \mathrm{q}$ is expressed in Kelvin by dividing the associated latent energy by the specific heat. The same applies to saturation humidity $\mathrm{q}^{*}$ below.
} 
The dry dynamics are similar to those used in previous studies, e.g. M00, MS01, KM06. The main new feature of this model is its convective parameterizations that determine $\mathrm{J}_{1}$ and $\mathrm{J}_{2}$, described below.

b) Convective parameterizations

First, we define an integrated upper tropospheric heating anomaly U (scaled by the depth of the troposphere)

$$
U=\left(J_{1}-J_{2}\right) / 2
$$

and an integrated lower tropospheric heating anomaly $\mathrm{L}$

$$
L=\left(J_{1}+J_{2}\right) / 2
$$

and assume that in statistical equilibrium, the ratio of the total upper tropospheric heating (mean plus anomaly) to the total lower tropospheric heating is related to the anomalous moisture deficit (relative to saturation) in the mid-troposphere by

$$
\frac{U+U_{0}}{L+L_{0}}=r_{0}+\frac{r_{q}}{L_{0}} q^{+}
$$

where the subscript 0 denotes background mean values, and $\mathrm{U}_{0}=\mathrm{r}_{0} \mathrm{~L}_{0}$, and a negative $\mathrm{q}^{+}$ indicates anomalous moisture deficit. In the mid-troposphere (say, 500hPa and 270K), upon expressing the saturation humidity $\mathrm{q}^{*}$ in units of Kelvin, we have $\partial \mathrm{q}^{*} / \partial \mathrm{T} \sim 1$. Further taking into account the fact that the first mode is near its peak value $(\pi / 2)$ in the midtroposphere, we have

$$
\begin{aligned}
& q^{+}=q_{\text {mid }}-\frac{\pi}{2} T_{1}\left(\frac{\partial q^{*}}{\partial T}\right)_{\text {midtrop }} \\
& \approx q_{\text {mid }}-1.5 T_{1}
\end{aligned}
$$


Eq. (10) states that for the same amount of convection in the lower troposphere, there is less convection in the upper troposphere when the mid-troposphere is dry. The notion that a dry mid-troposphere limits the depth of tropical convection is well supported by observations, numerical simulations, and theoretical reasoning (Brown and Zhang, 1997;Sherwood, 1999;Parsons et al., 2000;Redelsperger et al., 2002;Ridout, 2002;Derbyshire et al., 2004;Takemi et al., 2004;Roca et al., 2005;Kuang and Bretherton, 2006). One plausible interpretation (e.g. Brown and Zhang, 1997; Derbyshire et al. 2004; Kuang and Bretherton, 2006) is that all else being equal, with a dry mid-troposphere, convection does not reach as high because entrainment of drier environmental air by the rising air parcels leads to more evaporative cooling, and hence negative buoyancy. More detailed studies, however, are needed to place this interpretation on a firmer footing. Moisture deficit, or saturation deficit, has been used as a control on convection before (Raymond, 2000), except in terms of precipitation instead of the height of convection. We shall consider the adjustment of the U/L ratio (denoted as $\mathrm{r}$ ) to moisture deficit to be instantaneous. One could take into consideration the finite response time of $r$ so that:

$$
\frac{\partial r}{\partial t}=\tau_{r}^{-1}\left(r_{e q}-r\right)
$$

where $r_{\mathrm{eq}}$ is the ratio anomaly that is in statistical equilibrium with its large-scale environment (Eq. (10)) and $\tau_{\mathrm{r}}$ is the adjustment time for $\mathrm{r}$ to approach that equilibrium.

We consider $\tau_{\mathrm{r}}$ to be of the same order as the time for convective updrafts to rise from the lower troposphere to the upper troposphere (hours or less). For $\tau_{\mathrm{r}}$ values in this range, inclusion of this adjustment process does not change the basic behavior of the model so 
we will leave it out in this paper. Note that $\tau_{\mathrm{r}}$ is not the relaxation time for moisture anomalies, which will be discussed in section 3 and is on the order of a day.

Second, we consider that saturation moist static energy averaged over a layer above the cloud base is in quasi-statistical equilibrium (QE) with the subcloud layer moist static energy. When the equilibrium is achieved instantaneously, we have

$$
\frac{\partial h_{b}}{\partial t}=\left\langle\frac{\partial h^{*}}{\partial t}\right\rangle
$$

The bracket on the right hand side denotes averaging over a layer above the cloud base. The variable $\mathrm{h}^{*}$ is the saturation moist static energy. We then rewrite Eq. (13) as

$$
\frac{\partial h_{b}}{\partial t}=F \frac{\partial}{\partial t}\left[f T_{1}+(1-f) T_{2}\right]
$$

where $F=\partial h^{*} / \partial T$. The factors $f$ and (1-f) are the relative weights of $T_{1}$ and $T_{2}$. We shall interpret $f$ as measuring the importance of undiluted parcels in the total convective mass flux. An $\mathrm{f}$ close to 1 implies that the convective mass flux is dominated by undiluted parcels, and Eq. (13) holds with $<\mathrm{h}^{*}>$ taken as an average over the whole troposphere. In this case, the approximate invariance of convective available potential energy (CAPE) is effectively used as a simplification for QE over the whole depth of the troposphere, as in e.g. Emanuel et al. (1994). An $\mathrm{f}$ close to 0 implies that the convective mass flux is dominated by heavily entraining parcels, and Eq. (13) holds with $<\mathrm{h} *>$ averaged over a shallow layer above the cloud base. In this case, Eq. (14) effectively assumes convective inhibition (CIN) (within the two-vertical mode framework) to be approximately invariant. This is known as the boundary layer quasi-equilibrium (BLQ) (Emanuel, 1995;Raymond, 1995). Our normative value for $\mathrm{f}$ is 0.5 where $<\mathrm{h}^{*}>$ may be viewed as an average over 
the lower half of the troposphere. To emphasize the instantaneous adjustment, we will refer to Eq. (14) (or Eq. (13)) as strict quasi-equilibrium (SQE), following Emanuel et al. (1994), where the word "strict" simply means that the adjustment is instantaneous.

For a given U/L ratio, we plug Eqs. (3) and (5) into Eq. (14) and make use of Eqs. (8), (9), and (10) to solve for the lower tropospheric heating in $\mathrm{SQE}$, denoted as $\mathrm{L}_{\mathrm{eq}}$ :

$$
L_{e q}=B^{-1}\left[A r_{q} q^{+}+f w_{1}+(1-f) w_{2}\right]
$$

where

$$
\begin{aligned}
& q^{+} \equiv q-1.5 T_{1} \\
& A \equiv 1-2 f+\frac{b_{2}-b_{1}}{F} \\
& B \equiv 1+\frac{b_{2}+b_{1}}{F}-A r_{0}
\end{aligned}
$$

Eq. (15) is a straightforward restatement of the SQE condition of Eq. (14), and the various terms have clear physical meanings: provided that A and B are positive, uplifting in the lower troposphere or a moist mid-troposphere increases convective heating in the lower troposphere in SQE. $\mathrm{L}_{\mathrm{eq}}$ can also be expressed in terms of $\partial \mathrm{T} / \partial \mathrm{t}$. This form will be used in section 3. In Eq. (16) and for the rest of the paper, we omit the subscript in $\mathrm{q}_{\mathrm{mid}}$ to simplify the notation. Taking into consideration the finite adjustment time to achieve QE, denoted as $\tau_{\mathrm{L}}$, we have:

$$
\frac{\partial L}{\partial t}=\tau_{L}^{-1}\left(L_{e q}-L\right)
$$


We expect $\tau_{\mathrm{L}}$ to be on the order of a few hours, the time for a few turnovers of shallow cumulus convection. From $\mathrm{L}$ and the $\mathrm{U} / \mathrm{L}$ ratio, $\mathrm{J}_{1}$ and $\mathrm{J}_{2}$ can be easily determined, completing our convective parameterization.

The present convective parameterization falls within the QE framework first introduced by Arakawa and Schubert (1974), which states that convection should be in a state of statistical equilibrium with the large-scale flow. In some previous simple models of the interaction between large-scale circulation and deep convection (e.g. Emanuel et al., 1994), the approximate invariance of convectively available potential energy (CAPE) is used as a simplification for SQE over the whole depth of the troposphere (i.e. $f=1$ ). As noted earlier, this emphasizes the role of undiluted parcels in deep convective mass flux, based on which CAPE is computed. Recent evidence, however, indicates that this is not a good simplification of SQE, at least in cases such as convectively coupled waves. Highresolution numerical studies show that undiluted parcels do not make a significant contribution to the overall convective mass flux (Khairoutdinov and Randall, 2006; Kuang and Bretherton, 2006). This is corroborated by the observed and simulated sensitivity of convection to tropospheric moisture (see discussion in relation to Eq. (10)).

In the present treatment, we are using the invariance of a shallow CAPE as a simplification of SQE over the lower half of the troposphere (with $f=1 / 2$ in Eq. (14)). The shallow CAPE measures the integrated buoyancy for undiluted parcels only up to the mid-troposphere. This is a simplification for the present simple model with only two vertical modes in the free troposphere. It by no means suggests that cloud parcels do not experience entrainment in the lower troposphere. However, the cumulative effect of entrainment is smaller in the lower troposphere because of the shorter distance traveled 
by the cloud parcels and their smaller moist static energy difference from the environment (for the regions that we are concerned with, the lower troposphere is taken to be always sufficiently moist so that its moist static energy is close to that of the cloud parcels). Therefore, neglecting the effect of entrainment and using Eq. (14) is a reasonable simplification for SQE over the lower half of the troposphere. We then use Eq. (10) to explicitly include the effect of entrainment on the convective mass flux that can reach from the lower to the upper half of the troposphere. In Eq. (10), we have neglected the role of the traditional CAPE (defined for undiluted parcels over the whole depth of the free troposphere). This reflects the view that the mass flux reaching the upper troposphere is in significantly diluted updrafts, and mid-tropospheric moisture deficit is the dominating factor that controls the depth of convection (through entrainment). The CAPE anomalies can become important when they are sufficiently negative so that the background CAPE is substantially consumed, and is a nonlinearity that can limit the growth of the waves. Further discussions of nonlinearity, however, will not be presented in this paper.

c. Linearized equations and normative parameter values

The system is linearized by replacing Eq. (10) with

$$
U=r_{0} L+r_{q} q^{+}
$$

The linearized model thus consists of the prognostic equations (3), (6), and (17), and the auxiliary equations (8), (9), (15), and (18).

Observed and simulated data from, for example, K07 and Haertel and Kiladis (2004), may be used to estimate the parameters by viewing such data in the framework of the 
present model. This is discussed in Appendix A. Table 1 lists the normative parameter values used in the paper based on these estimates. It is important to stress that because of the highly simplified nature of the present model, viewing observations or the CSRM results in its framework is very approximate and the parameter estimates are only intended as educated guesses of plausible values.

\section{d. Linear Analysis}

Results from a linear analysis of this system are shown in Figure 1. Waves with wavelengths from $1500 \mathrm{~km}$ to $40000 \mathrm{~km}$ are unstable with a maximum growth rate of about $0.13 /$ day at $5000 \mathrm{~km}$. The unstable waves have phase speeds around $20 \mathrm{~m} / \mathrm{s}$, slightly slower than the dry wave speed of the second vertical mode. The eigenvector at a wavelength of $8640 \mathrm{~km}$ is expressed in physical space in Figure 2a,b. The eigenvector is scaled so that $\mathrm{T}_{1}$ is a sine function with amplitude of one. We have further reconstructed the vertical structure of temperature and convective heating (Figure $2 \mathrm{c}, \mathrm{d}$ ) so that it is visually more direct to compare with observations. We have used $\sin \left(\mathrm{j} \pi \mathrm{z} / \mathrm{H}_{\mathrm{T}}\right), \mathrm{j}=1,2$ as the vertical structures with $\mathrm{H}_{\mathrm{T}}=14 \mathrm{~km}$. These figures show that the linear model yields instability at wavelengths and with structures that compare well with the CSRM simulations and observations. Note that convective heating here is dominated by the first vertical mode and has a significant tilt, consistent with the observations/ simulations (Haretel and Kiladis, 2004; K07). In contrast, in the model of KM06, heating in the upper troposphere is substantially stronger than that in the lower troposphere (their Fig. 6).

There is also a tendency for this to be true in MS01 as the wavelength increases to beyond 2000km (their Fig. 4). The substantially stronger heating in the upper troposphere indicates a larger contribution from the second mode heating and a more in-phase relation 
between $J_{1}$ and $-J_{2}$, compared to Figure 2 d. Figure 3 shows the phase lag between $J_{1}$ and $-\mathrm{J}_{2}$ as a function of wavenumber. Substantial phase lag is seen at all wavenumbers. Over a wide range of wavelengths (from $1500 \mathrm{~km}$ to $10000 \mathrm{~km}$ ), the phase lag is between $\sim 85$ to $\sim 65$ degrees. This is consistent with the CSRM simulation results from K07.

We have repeated the linear analysis with the parameters perturbed around their normative values one at a time. The parameter dependence of the maximum linear growth rate is shown in Figure 4. While not shown, the phase speeds of the most unstable modes are between 10 and $25 \mathrm{~m} / \mathrm{s}$ except with $\mathrm{b}_{1}<0.6, \mathrm{~b}_{2}>3.3, \mathrm{~d}_{1}<0.9, \mathrm{~d}_{2}>-0.6, \mathrm{a}_{1}>1.6$, or $\mathrm{a}_{2}<-0.4$, where the phase speeds of the most unstable modes are a few $\mathrm{m} / \mathrm{s}$ or less. While it is useful to know the parameter sensitivities of the model, for the purpose of revealing the basic instability mechanisms, it is more informative to consider certain limiting cases, as discussed in section 3 .

It is useful to note that while $\mathrm{r}_{\mathrm{q}}$ and $\mathrm{f}$ are separate parameters in the model and are varied independently in Figure 4, they are related to the conceptual picture of whether upper troposphere convective mass flux is dominated by nearly undiluted or significantly diluted updrafts. When significantly diluted updrafts dominate, the mid-troposphere humidity has an important effect on the depth of convection, and $r_{q}$ will be large, $f$ will be small (a smaller depth of the atmosphere can be assumed to be in QE regardless of the environment humidity). Thus one should in principle vary these parameters together to be conceptually consistent, although this is not done here.

\section{The basic instability mechanisms}

3.a A slightly simplified version of the model 
To reveal its basic instability mechanisms, we make a few simplifications to the model described in section 2. First, we replace the two-way wave equations Eq. (3) with oneway wave equations with a Newtonian cooling coefficient $\varepsilon$ :

$$
\frac{\partial T_{j}}{\partial t}+c_{j} \frac{\partial T_{j}}{\partial x}=J_{j}-\varepsilon T_{j}
$$

and use Eq.(7) instead of Eq.(6) to evolve mid-tropospheric humidity. Replacing the twoway wave equations by one-way wave equations has some quantitative effects, as discussed in Appendix B. For example, heating drives temperature anomalies more effectively in the one-way wave equations, and this effect is stronger for the first vertical mode. However, these changes do not alter the basic behavior of the model. It is also convenient to rewrite Eq.(18) in terms of $\mathrm{J}_{1}$ and $\mathrm{J}_{2}$ :

$$
J_{2}=\gamma_{0} J_{1}-\gamma_{q} q^{+}
$$

where

$$
\begin{gathered}
\gamma_{0} \equiv \frac{1-r_{0}}{1+r_{0}} \\
\gamma_{q} \equiv \frac{2 r_{q}}{1+r_{0}}
\end{gathered}
$$

and include the finite adjustment time to achieve $\mathrm{QE}$ as:

$$
\frac{\partial J_{1}}{\partial t}=\tau_{J}^{-1}\left(J_{1, e q}-J_{1}\right)
$$

where

$$
J_{1, e q}=\frac{1}{b_{1}+b_{2} \gamma_{0}}\left\{b_{2} \gamma_{q} q^{+}-F \frac{\partial}{\partial t}\left[f T_{1}+(1-f) T_{2}\right]\right\}
$$


Here, we have expressed $\mathrm{J}_{1, \mathrm{eq}}$ in terms of $\partial \mathrm{T} / \partial \mathrm{t}$, although one can also write it in a form similar to Eq. (15). We shall take $\tau_{\mathrm{J}}=\tau_{\mathrm{L}}$. Physically, it is perhaps more natural to apply the finite adjustment time on lower tropospheric heating $\mathrm{L}$ instead of $\mathrm{J}_{1}$, as $\mathrm{L}$ is more locally determined by the subcloud layer and the lower troposphere. However, since $\mathrm{J}_{1}=\left(1+\mathrm{r}_{0}\right) \mathrm{L}+\mathrm{r}_{\mathrm{q}} \mathrm{q}^{+}$, the difference in relaxing $\mathrm{J}_{1}$ instead of $\mathrm{L}$ lies in the $\partial \mathrm{q}^{+} / \partial \mathrm{t}$ term. Because $\tau_{\mathrm{L}}$ is considerably shorter than the timescale for $\mathrm{q}^{+}$to vary (on the order of the wave period), Eq. (22) has the same basic effect as Eq. (17).

The phase speeds and the linear growth rates for this simplified version are shown in Figure 5 and the structures for a wavelength of $8640 \mathrm{~km}$ are shown in Figure 6 . The higher wavenumbers are more stable compared to Figure 1 and the amplitudes of $\mathrm{J}_{1}$ and $\mathrm{J}_{2}$ are smaller compared to those in Figure 2. The latter is because heating is more effective in driving temperature anomalies in one-way equations (Appendix B). When this difference is accounted for following discussion in Appendix B, the amplitudes of $\mathrm{J}_{1}$ and $\mathrm{J}_{2}$ become similar to those in Figure 2. We have also repeated the linear analysis with the parameters perturbed around their normative values one at a time for this simplified version of the model. The parameter dependence of the maximum linear growth rate is shown in Figure 7, and is similar to that in Figure 4 in terms of its basic pattern. The parameter dependence on $\mathrm{m}_{1}$ and $\mathrm{m}_{2}$ are shown instead of $\mathrm{a}_{1}, \mathrm{a}_{2}, \mathrm{~d}_{1}$, and $\mathrm{d}_{2}$ because Eq (7) is used. The dependence on $\varepsilon$ is also similar to that in Figure 4 (not shown). Similar to the model in section 2, all unstable waves have phase speeds between 10 and $25 \mathrm{~m} / \mathrm{s}$ except with $b_{1}<0.6, b_{2}>10 / 3, m_{1}>0.5$, or $m_{2}<0.6$, where the phase speeds of the most unstable modes are a few $\mathrm{m} / \mathrm{s}$ or less. This dependence is explained in section 3.b.2 and Appendix 
C. The similarity in the basic parameter dependence indicates that the simplified system captures the basic behavior of the model described in section 2c.

In this paper, we focus on regimes with $m_{1}>0$. Moistening of the mid-troposphere by deep convection is clearly seen in $\mathrm{K} 07$. With $\mathrm{m}_{1}>0$, the basic behavior of the system is preserved without including contribution of $\mathrm{T}_{1}$ in $\mathrm{q}^{+}$so we will take $\mathrm{q}^{+}=\mathrm{q}$. The contribution of $\mathrm{T}_{1}$ in $\mathrm{q}^{+}$has an important stabilizing role when $\mathrm{m}_{1}<0$. This regime, however, is not the subject of this paper, and will not be discussed further.

3.b The regime with $\gamma_{0} \geq 0$ and a moisture stratiform instability

We first consider the model behavior with $\gamma_{0} \geq 0$ (i.e. $r_{0} \leq 1$ ), which corresponds to an atmosphere in which, climatologically speaking, the convective heating in the lower troposphere is greater than or equal to that in the upper troposphere. We shall try to identify the model's basic instabilities by considering limiting cases. To simplify the discussion, we take $b_{2}=0$ and $\gamma_{0}=0$. Physically, $b_{2}=0$ means that convection in the upper and lower troposphere have the same effect on $h_{b}$ per unit heating and $\gamma_{0}=0$ means that the background convective heating is of the same strength in the upper and lower troposphere. These are not required but help to simplify our discussion. The results are largely representative of general cases with $\gamma_{0} \geq 0$ and non-representative results will be pointed out along the way. Limiting cases with general parameter choices can be reduced to the same form by redefining the parameters as discussed in Appendix C.

\section{3.b.1. Limiting case $\mathrm{I}: \mathrm{f}=1$}

Let us first consider the limiting case with $\mathrm{f}=1$ so that Eq. (23) reduces to 


$$
J_{1, e q}=-\frac{F}{b_{1}} \frac{\partial T_{1}}{\partial t}
$$

Physically, this represents a case where convective mass flux is dominated by undiluted updrafts. In this case the sub-cloud layer moist static energy $h_{b}$ is changed only by $J_{1}$ (as $\mathrm{b}_{2}=0$ ) and is in equilibrium with $\mathrm{T}_{1}$ alone (as $\mathrm{f}=1$ ). Eq. (24) simply states that $\mathrm{J}_{1, \mathrm{eq}}$ is that required to keep $\partial \mathrm{h}_{\mathrm{b}} / \partial \mathrm{t}$ the same as $\mathrm{F} \partial \mathrm{T}_{1} / \partial \mathrm{t}$. In this case, the first mode (temperature and heating) is no longer affected by the second mode or moisture. This reduces the system to the first baroclinic mode model discussed in previous studies (Emanuel, 1987; Neelin et al., 1987). Let us start with $\varepsilon=0$ (no Newtonian cooling) and $\tau_{\mathrm{J}}=0$ (i.e. in SQE), so that

$$
\frac{\partial T_{1}}{\partial t}+c_{1} \frac{\partial T_{1}}{\partial x}=-\frac{F}{b_{1}} \frac{\partial T_{1}}{\partial t}
$$

The convectively coupled first vertical mode has a zero growth rate and a phase speed $\mathrm{c}_{1}{ }^{*}=\mathrm{c}_{1} /\left(1+\mathrm{F} / \mathrm{b}_{1}\right)$ due to the reduced effective static stability. The first vertical mode also forces a response in $T_{2}$ and $q$ (in a one-way interaction) through the effect of $\mathrm{J}_{1}$ on $\mathrm{J}_{2}$, both directly (Eq.(20)), and indirectly through $\mathrm{J}_{1}$ 's effect on moisture, and resonance occurs when $\mathrm{c}_{2}=\mathrm{c}_{1}{ }^{*}$. The direct effect vanishes with $\gamma_{0}=0$, but does not with more general parameter choices. The temperature and heating structures for a simple case $\left(b_{2}=0, \gamma_{0}=0\right.$, $\varepsilon=0, \tau_{\mathrm{J}}=0$, and $\mathrm{m}_{2}=0$; other parameters take their normative values) are shown in Figure 8. The $\mathrm{q}$ field in this case is simply $-\mathrm{J}_{2} / \gamma_{\mathrm{q}}$. The phase speed $\mathrm{c}_{1}{ }^{*}$ is $10 \mathrm{~m} / \mathrm{s}$ and the wave structures in many aspects resemble the observed patterns.

So far, the growth rate is 0 for all wavenumbers. Introducing a finite $\tau_{\mathrm{J}}$ causes heating $\mathrm{J}_{1}$ to lag $\mathrm{T}_{1}$ by more than $\pi / 2$ in phase, which gives rise to a damping effect that is stronger 
at high wavenumbers. This has been pointed out before and was named moist convective damping (MCD) (Emanuel, 1993;Emanuel et al., 1994;Neelin and Yu, 1994;Yu and Neelin, 1994). A positive $\varepsilon$ further damps the waves.

\section{3.b.2. Limiting case II: $\mathrm{f}=0$}

Let us now consider $\mathrm{f}=0$, corresponding to an atmosphere in which heavily entraining parcels dominate the convective mass flux. Here, the sub-cloud layer $h_{b}$ is in equilibrium with $\mathrm{T}_{2}$ alone, and there is no more dependence on $\mathrm{T}_{1}$ of the other variables. Again, we take $b_{2}=0$ and $\gamma_{0}=0$ for simplicity. In this case, Eq. (23) reduces to

$$
J_{1}=-\frac{F}{b_{1}} \frac{\partial T_{2}}{\partial t}
$$

i.e., $\mathrm{J}_{1}$ is that required to keep $\partial \mathrm{h}_{\mathrm{b}} / \partial \mathrm{t}$ the same as $\mathrm{F} \mathrm{T}_{2} / \partial \mathrm{t}$. First consider a system in $\mathrm{SQE}$,

$$
\begin{gathered}
\frac{\partial T_{2}}{\partial t}+c_{2} \frac{\partial T_{2}}{\partial x}=-\gamma_{q} q \\
\frac{\partial q}{\partial t}=-m_{1} \frac{F}{b_{1}} \frac{\partial T_{2}}{\partial t}-m_{2} \gamma_{q} q
\end{gathered}
$$

where we have used $J_{2}=-\gamma_{q} q\left(\right.$ as $\left.\gamma_{0}=0\right)$. Assuming solutions of the form $\exp [i(k x-\omega t)]$, we obtain the dispersion relationship

$$
\omega^{2}-\left[c_{2} k+i \gamma_{q}\left(\frac{F m_{1}}{b_{1}}-m_{2}\right)\right] \omega-i m_{2} \gamma_{q} c_{2} k=0
$$

Eqs. (27) and (28) describe a coupled system of $\mathrm{T}_{2}$ and q. The effect of $\mathrm{T}_{2}$ on $\mathrm{q}$ (through its effect on $\mathrm{J}_{1}$ and, in turn, the moistening effect of $\mathrm{J}_{1}$ on $\mathrm{q}$, as expressed in Eq.(28)) coupled with the effect of $q$ on $\mathrm{T}_{2}$ (through its effect on $\mathrm{J}_{2}$ and, in turn, the heating effect of $\mathrm{J}_{2}$ on $\mathrm{T}_{2}$, as expressed in Eq. (27)) give rise to an instability. This is best seen with 
$\mathrm{m}_{2}=0$. Putting aside the less interesting solution $\omega=0$, we have $\mathrm{q}=-\left(\mathrm{m}_{1} \mathrm{~F} / \mathrm{b}_{1}\right) \mathrm{T}_{2}$ and the heating $J_{2}$ is exactly in phase with $T_{2}$ and $\omega=c_{2} k+i m_{1} \gamma_{9} F / b_{1}$, i.e. the phase speed is $c_{2}$ and the growth rate is $m_{1} \gamma_{q} \mathrm{~F} / \mathrm{b}_{1}$ at all wavelengths. The growth rate is proportional to $m_{1} \gamma_{q}$, which measures how strongly the depth of convection depends on $\mathrm{q}$ (the factor $\gamma_{\mathrm{q}}$ ) and how strongly moisture depends on $\mathrm{J}_{1}$ (the factor $\mathrm{m}_{1}$ ). It is also proportional to $\mathrm{F} / \mathrm{b}_{1}$, which measures how strongly $\mathrm{J}_{1}$ depends on $\partial \mathrm{T}_{2} / \partial \mathrm{t}$. While we have chosen $\mathrm{b}_{2}=0$ and $\gamma_{0}=0$ here, this picture holds for more general parameter choices as well (Appendix C), except the strict dependence on $m_{1}$, which is specific to the choice of $b_{2}=0, \gamma_{0}=0$. For more general parameter choices, a modified $\mathrm{m}_{1}, \hat{m}_{1} \equiv \frac{m_{1}+m_{2} \gamma_{0}}{1+b_{2} \gamma_{0} / b_{1}}$, should be used (Appendix C).

In this limiting case, $T_{1}$ is forced by $J_{1}$ but does not feed back onto $J_{1}$. The physical structure of the wave with a wavelength of $8640 \mathrm{~km}$ from this limiting case in SQE is shown in Figure 9. The q field in this case is again simply $-\mathrm{J}_{2} / \gamma_{\mathrm{q}}$.

A positive $m_{2}$, which implies that the net effect of the second mode heating is to moisten the troposphere, brings a damping effect on q (Eq. (28)). The physical picture is simple: when, for instance, the troposphere is dry $(\mathrm{q}<0)$, convection is shallower $\left(\mathrm{J}_{2}>0\right)$; the combined effect of vertical advection and precipitation associated with the second mode heating moistens the atmosphere (i.e. $\mathrm{m}_{2} \mathrm{~J}_{2}>0$ ), reducing the dry anomaly. That $\mathrm{m}_{2}$ is positive should be expected based on observations: the effect of second mode vertical advection on mid-troposphere moisture is small; more shallow convection and less stratiform precipitation reduce the removal of moisture by precipitation. The factor $\mathrm{m}_{2} \gamma_{\mathrm{q}}$ defines a relaxation timescale for moisture anomalies when the $\partial \mathrm{T}_{2} / \partial \mathrm{t}$ term in Eq. (28) 
vanishes. For our normative parameter choices, the relaxation timescale is about a day and half.

With a nozero $\partial T_{2} / \partial t$ term in Eq. (28), moisture is coupled to $T_{2}$. In this case, a relaxation timescale for moisture alone is not defined, and the $-\mathrm{m}_{2} \gamma_{\mathrm{q}} \mathrm{q}$ term acts to reduce the growth rate of the unstable mode. This effect is stronger at lower frequencies, i.e. the moistening effect of $\mathrm{J}_{2}$ acts to preferentially damp low wavenumbers. More quantitatively, consider small departures in $\omega$ from its value for $m_{2}=0$, i.e. $\omega=\delta \omega+c_{2} k+i m_{1} \gamma_{q} F / b_{1}$. Plugging this into (29) and ignoring second order terms of $\delta \omega$, we have

$$
\delta \omega \approx \frac{m_{1} m_{2}\left(F / b_{1}\right) \gamma_{q}^{2}}{c_{2} k+i\left(m_{2}+m_{1} F / b_{1}\right) \gamma_{q}}
$$

Therefore, this damping effect becomes significant for wavenumbers lower than $\left(m_{2}+m_{1} F / b_{1}\right) \gamma_{q} / c_{2}$. With the normative parameter values, this corresponds to a wavelength of $9000 \mathrm{~km}$. The effect of $m_{2}$ on the growth rate is shown in Figure 10 (thin solid line). With $\mathrm{m}_{2}<0$, Eq. (28) contains a unstable moisture mode; without the $\partial \mathrm{T}_{2} / \partial \mathrm{t}$ term, it has zero phase speed and a growth rate of $\mathrm{m}_{2} \gamma_{\mathrm{q}}$. For general parameter choices, an effective $\mathrm{m}_{2} \hat{m}_{2} \equiv \frac{b_{1} m_{2}-b_{2} m_{1}}{b_{1}+b_{2} \gamma_{0}}$ should be used and the effective $\mathrm{m}_{2}$ becomes negative when $b_{1}<0.6, b_{2}>10 / 3, m_{1}>0.5$, or $m_{2}<0.6$, which are unstable regimes in Figure 7 with very small phase speeds. These modes are therefore attributed to a moisture instability described by Eq. (28) with a negative (effective) $\mathrm{m}_{2}$. These modes are similar to the standing modes found in KM06 (their Fig. 4a).

Departing from SQE by introducing a finite response time, i.e. replacing Eq. (28) by 


$$
\begin{aligned}
& \frac{\partial q}{\partial t}=-m_{1} J_{1}-m_{2} \gamma_{q} q \\
& \frac{\partial J_{1}}{\partial t}=\tau_{J}^{-1}\left(-\frac{F}{b_{1}} \frac{\partial T_{2}}{\partial t}-J_{1}\right)
\end{aligned}
$$

shifts $J_{2}$ out of phase with $T_{2}$ and reduces the growth rate. This is similar to the MCD effect discussed in the $\mathrm{f}=1$ case and is stronger for higher wavenumbers. Figure 10 shows the growth rate with the effect of $\tau_{\mathrm{J}}$ included (dotted line) and with the effects of both $\mathrm{m}_{2}$ and $\tau_{\mathrm{J}}$ included (diamond symbol). The mathematical reason for the different effects of $\mathrm{m}_{2}$ and $\tau_{\mathrm{J}}$ is simply that the latter involves $\partial / \partial \mathrm{t}$ and the former does not.

The above discussion paints the following physical picture for the basic instability in the limiting case II: Start with SQE, zero net moistening from the second mode heating $\left(\mathrm{m}_{2}=0\right)$ and no dissipation $(\varepsilon=0)$, and with the propagation of a second vertical mode temperature anomaly $\mathrm{T}_{2}$. The $\mathrm{T}_{2}$ anomaly modulates deep (or first baroclinic) convective heating $\mathrm{J}_{1}$ by perturbing the statistical equilibrium between the lower troposphere and the subcloud layer. The result is a $\mathrm{J}_{1}$ field that lags $\mathrm{T}_{2}$ by $90^{\circ}$ in phase. This changes the moisture field, which lags $\mathrm{J}_{1}$ by another $90^{\circ}$ and is therefore $180^{\circ}$ out of phase with $\mathrm{T}_{2}$. As $J_{2}=-\gamma_{\mathrm{q}} \mathrm{q}$, it is in phase with $T_{2}$ and causes growth. This basic instability is illustrated schematically in Figure 11 and will be referred to as the moisture-stratiform instability. While an eastward propagating wave is chosen for the illustration, the same instability mechanism also operates in westward propagating or standing waves. In the case of a standing wave, the phase lag will be manifested as a lag in time. Note that while we have used the name "stratiform" following M00, it is intended here to represent both phases of $\mathrm{J}_{2}$, i.e., both stratiform and shallow/congestus convection. Building upon this basic 
instability, we now add the moistening effect of $\mathrm{J}_{2}$, which reduces growth rates more strongly at low wavenumbers, and the finite time to approach $\mathrm{QE}$, which reduces growth rates more strongly at high wavenumbers, and the dissipation $\varepsilon$, which damps the waves more or less uniformly in wavenumber. These damping mechanisms shape the otherwise uniform growth rate curve to favor wavelengths of a few thousand kilometers (circles in Figure 10).

3.b.3. Basic difference between the two limiting cases

The following steps shown in Figure 11 are the same in both limiting cases: (b) $\rightarrow$ (c) $\rightarrow$ (d) $\rightarrow$ (a). The main difference between the two is that in the limiting case II with $\mathrm{f}=0$, the second baroclinic temperature anomaly controls the first baroclinic heating, and the feedback loop illustrated in Figure 11 is complete, giving rise to the moisturestratiform instability, while in the limiting case I with $\mathrm{f}=1$, the first baroclinic temperature anomaly controls the first baroclinic heating, the feedback loop is not complete, and all waves are stable. The basic reason for the different behavior of the two vertical modes is that the first baroclinic heating $\mathrm{J}_{1}$ is more strongly tied to $\partial \mathrm{T} / \partial \mathrm{t}$ (controlled by shallow or deep CAPE) while the second baroclinic heating $\mathrm{J}_{2}$ is more strongly tied to moisture (moisture control). This allows $J_{2}$ to be more in phase with $T_{2}$ when $J_{1}$ is controlled by $T_{2}$, but constrains $J_{1}$ to be largely in quadrature with $T_{1}$ when it is controlled by $T_{1}$. While we have taken $b_{2}=0, \gamma_{0}=0$ for conceptual simplicity, the same conclusion can be drawn with more general parameter choices, as discussed in Appendix C.

3.b.4. Intermediate cases: $0<f<1$ 
We are unaware of general mathematical results that relate intermediate cases to the two limiting cases in a simple and physically meaningful way. We have therefore examined the model behavior for intermediate values of $\mathrm{f}$ empirically. The moisture stratiform instability remains the basic instability (all waves are stable when either $\mathrm{m}_{1}$ or $\gamma_{\mathrm{q}}$ is zero), and the moistening effect of $\mathrm{J}_{2}$ and the MCD effect continue to shape the growth rate curve by reducing the growth rates, more strongly at low and high wavenumbers, respectively. Although, even with $\mathrm{m}_{2}=0$, as f increases, the instability is reduced more strongly at low wavenumbers (Figure 12), indicating the presence of other stabilization effects at low wavenumbers in addition to the moistening effect of $\mathrm{J}_{2}$. There is enhanced instability near $\mathrm{f} \sim 0.2$ particularly at higher wavenumbers. This is associated with the resonance effect present in the limiting case I (when $\mathrm{c}_{2} \sim \mathrm{c}_{1} *$ ). The larger resonance effects at higher wavenumbers can be understood in mathematical terms. However, given the highly idealized nature of the present model and the strong MCD damping effect at high wavenumbers, the relevance of such resonance to the real atmosphere is not clear.

3.c The case of $\gamma_{0}<0, \gamma_{\mathrm{q}}=0$ and the stratiform instability of Mapes (2000)

As seen in Figure $7 d$ (and Figure 4d), there is a branch of unstable waves with $\mathrm{r}_{0}>1$ (or $\gamma_{0}<0$ ) that behaves differently from that with $r_{0} \leq 1$ (or $\gamma_{0} \geq 0$ ). This represents a case where the background mean convective heating is stronger in the upper troposphere. Its behavior is best exposed by setting $\gamma_{\mathrm{q}}=0$. To simplify the discussion, we will also take $\mathrm{b}_{2}=0, \varepsilon=0$, and $\tau_{\mathrm{J}}=0$, although these are not required. The system is now reduced to: 


$$
\begin{aligned}
& \frac{\partial T_{1}}{\partial t}+c_{1} \frac{\partial T_{1}}{\partial x}=J_{1, e q} \\
& \frac{\partial T_{2}}{\partial t}+c_{2} \frac{\partial T_{2}}{\partial x}=\gamma_{0} J_{1, e q} \\
& J_{1, e q}=-f \frac{F}{b_{1}} \frac{\partial T_{1}}{\partial t}-(1-f) \frac{F}{b_{1}} \frac{\partial T_{2}}{\partial t}
\end{aligned}
$$

Figure 13a shows that this simple system qualitatively reproduces the $r_{0}>1$ branch of the unstable modes seen in Figure 7d and Figure 4d. The large growth rates in Figure 13a, particularly those at high wavenumbers, are reduced and come to closer agreement with Figure $7 \mathrm{~d}$ when an adjustment time to $\mathrm{QE}\left(\tau_{\mathrm{J}}=2 \mathrm{hr}\right.$ ) is included (Figure $\left.13 \mathrm{~b}\right)$.

The dispersion relation for this system is quadratic and can be readily derived. It is easy to show analytically that a necessary condition for instability is $\gamma_{0}<0$ and that the growth rate is proportional to wavenumber. Therefore, this mechanism depends on positive stratiform heating (negative $J_{2}$ ) being tied to positive deep convective heating $\left(J_{1}\right)$ directly through $\gamma_{0}$ being less than 0 instead of indirectly through $\mathrm{J}_{1}$ 's effect on $\mathrm{q}$. We shall refer to this as the direct stratiform instability mechanism to distinguish it from the moisturestratiform instability mechanism discussed in section 3.b.

An example of the structure of the unstable waves from Eq. (32) with $\mathrm{f}=0.5$ and $\gamma_{0}=-0.2$ is shown in Figure 14. With $\mathrm{f}=0.5$, the phase and amplitude relationship between $\mathrm{T}_{1}$ and $T_{2}$ is such that $\mathrm{J}_{1}$, which is proportional to $\partial\left(T_{1}+T_{2}\right) / \partial t$, is roughly in opposite phase with $T_{2}$. This sets up a feedback loop that is the same as that of $M 00$ : when $T_{2}$ is negative (cold below and warm above), deep convection is enhanced; with $\gamma_{0}<0$, a negative $\mathrm{J}_{2}$ (cooling below and heating above) is tied to enhanced deep convection (positive $\mathrm{J}_{1}$ ), and amplifies the $T_{2}$ anomaly. Therefore, the basic instability mechanism in this regime is 
identified to be the same as the stratiform instability of M00. The wave structure is affected by replacing two-way wave equations with one-way wave equations. While not shown, when the two-way wave equations are used with $\mathrm{r}_{0}=1.5$ (i.e. $\gamma_{0}=-0.2$ ) and $\mathrm{r}_{\mathrm{q}}=0$, the temperature structure of the wave captures the salient features of the observed waves quite well.

The formulation in Eq. (32) is indeed similar to that of M00 and MS01, except that M00 has an additional prognostic equation for the subgrid-scale triggering energy. The effects of $T_{2}$ and $T_{1}$ on $\mathrm{J}_{1}$ are similar to their roles in the CAPE calculation in M00 and MS01 except that here $\mathrm{J}_{1}$ is determined implicitly from $\partial \mathrm{T} / \partial \mathrm{t}$ based on the QE concept instead of the explicit prognostic approach of M00 and MS01. As discussed in section 2b, the relative importance of $\partial T_{2} / \partial t$ and $\partial T_{1} / \partial t$ in determining $J_{1}$, as measured by the parameter $\mathrm{f}$, indicates the depth of the troposphere that is in QE regardless of the environmental humidity, and is physically interpreted as the importance of undiluted parcels in the convective mass flux. A similar interpretation can be made for the role of $\mathrm{T}_{2}$ in the CAPE calculation in MS01 as this includes the effect of entrainment. Values used in MS01 and KM06 imply an $f$ of 0.9 . The relative weights of $T_{1}$ and $T_{2}$ in the CAPE calculation in M00 imply an $\mathrm{f}$ value of 0.8 , although the role of $\mathrm{T}_{2}$ on convection is further enhanced by its role in his CIN calculation, which would imply an $f$ value of $\sim 0.1-0.2$. The effective $f$ in M00 therefore varies between 0.1 to 0.8 , depending on how strong the CIN control is relative to the CAPE control, and can be close to our normative value.

The system of Eq. (32) constrains $J_{2}$ to be in opposite phase with $J_{1}$, so there is no tilted heating structure as in observations. The tilt can be introduced with a time lag between $\mathrm{J}_{2}$ and $\mathrm{J}_{1}$ : 


$$
\frac{\partial J_{2}}{\partial t}=\tau_{J 2}^{-1}\left(\gamma_{0} J_{1}-J_{2}\right)
$$

as done in M00, where a $3 \mathrm{hr}$ time lag is used, representing the time lag between the stratiform phase and the convective phase of a mesoscale convective system (MCS). This, however, provides appreciable tilts only at short wavelengths; a $3 \mathrm{hr}$ time lag corresponds to only $270 \mathrm{~km}$ with a wave speed of $25 \mathrm{~m} / \mathrm{s}$. For a wavelength of $864 \mathrm{~km}$, for example, and a $3 \mathrm{hr}$ time lag, $\mathrm{J}_{2}$ lags $-\mathrm{J}_{1}$ by $\sim 40^{\circ}$ and appreciable tilt is indeed seen in the heating structure. However, for longer wavelengths (for example, the case with a wavelength of $8640 \mathrm{~km}$ shown in Figure 14$)$, the same time lag produces little tilt $\left(\mathrm{J}_{2}\right.$ lags $-\mathrm{J}_{1}$ by $\sim 8^{\circ}$ ), as one should expect from Eq. (33). Indications of this behavior are also evident in MS01 (e.g. their Fig.4). Therefore, it is difficult for the basic instability mechanism in this regime $\left(\gamma_{0}<0\right.$ and $\left.\gamma_{q}=0\right)$ to produce the significant tilt seen in the observed heating field of large-scale waves. While we have taken $b_{2}=0, \varepsilon=0$, and $\tau_{\mathrm{J}}=0$ to simplify the discussion, the basic results remain the same without these simplifications. Eq. (33) with an adjustment time of $3 \mathrm{hr}$ also has the effect of further reducing the growth rates at high wavenumbers, and selecting waves of synoptic scale $(\sim 2000 \mathrm{~km}$ in wavelength) as the fastest growing (not shown).

As discussed in section 2.b, we have neglected the role of undiluted CAPE on the depth of convection based on the view that all updrafts experience significant entrainment and midtropospheric moisture deficit is the main factor affecting the depth of convection. In this section, as we have set $\gamma_{\mathrm{q}}=0$, one may wish to include the effect of undiluted CAPE and replace Eq. (20) with

$$
J_{2}=\gamma_{0} J_{1}-\gamma_{T} T
$$


which changes the second equation in (32) to

$$
\frac{\partial T_{2}}{\partial t}+c_{2} \frac{\partial T_{2}}{\partial x}=\gamma_{0} J_{1, e q}-\gamma_{T} T_{2}
$$

This adds a simple damping effect on $\mathrm{T}_{2}$, which is the same as the cumulus congestus damping effect on the second mode temperature in section $2 \mathrm{~b} 3$ of M00.

3.d Moisture-stratiform instability versus direct stratiform instability

From the CSRM simulations of K07, which are idealized simulations based on the Tropical Ocean Global Atmosphere Coupled Ocean-Atmosphere Response Experiment (TOGA-COARE) (Webster and Lukas, 1992), there are two pieces of evidence against the direct-stratiform instability being the main instability. First, when vertical advection of moisture is disabled in K07, convectively coupled waves are largely suppressed, while the direct-stratiform instability mechanism is not affected by the removal of vertical moisture advection. Second, substantial tilt in the heating structure is found in K07 across a wide range of wavenumbers. This is also inconsistent with the direct-stratiform instability mechanism. When stratiform heating is tied to deep convective heating with a fixed time lag based on the life cycle of MCSs, the tilt in the heating structure is expected to become increasingly small as wavelength increases. This is noted by Mapes et al. (2006). In contrast, the moisture-stratiform instability requires the vertical advection of moisture and yields substantial tilt in the convective heating structure over a wide range of wavenumbers (Figure 3). Indeed, in its limiting form, Eqs.(27) and (28), $\mathrm{J}_{2}$ lags $-\mathrm{J}_{1}$ by a quarter cycle. The moisture-stratiform instability is therefore more in line with the CSRM simulations of $\mathrm{K} 07$, and is suggested as the main instability mechanism in these simulations, and likely in the real atmosphere under TOGA-COARE conditions as well. 
Whether and how this might change with the background mean state is a subject of interest and warrants further research.

\section{Summary and discussion}

In this paper, we have developed a toy model of convectively coupled waves. Its main new feature is a conceptually simple treatment of convection based on the quasiequilibrium concept, simplified for a model of crude vertical structure. For convection in the lower troposphere we neglect the effect of entrainment and for convection reaching the upper troposphere, we emphasize the effect of entrainment and thus the impact of environmental moisture deficit. For realistic model parameters based on the results of CSRM simulations of K07, the toy model produces unstable waves at wavelengths and with structures that compare reasonably well with the CSRM results.

It is of interest to contrast the present treatment with that of M00, which is an influential model of convectively coupled waves. M00 introduced his model by raising the question: "if CIN is in equilibrium with a statistically ubiquitous population of small entraining cumuli, then how can it be a significant factor inhibiting deep-convective cells, which should presumably suffer less entrainment due to their larger size?" This led to his separate treatments for shallow and deep convection, where the role of shallow convection is a simple damping effect on the second mode temperature anomaly, and the effect of inhibition and triggering is emphasized through the effect of convective inhibition (CIN) and subcloud layer kinetic energy (or triggering energy) on the deep convective mass flux. This separate treatment, however, does not resolve the inconsistency raised by M00, and was recognized as a conceptual deficiency by M00, as shallow and deep convection are obviously interrelated. This inconsistency is absent in 
our treatment, where CIN is indeed not a significant factor in inhibiting deep-convective cells; instead, mid-troposphere moisture deficit is the main factor. We also eliminate triggering and inhibition from the conceptual picture and consider convection to be in quasi-equilibrium with the large-scale flow. Triggering and inhibition do occur; however, they reflect more of a view on individual storm scale instead of that on a large scale. On a large scale, we maintain that a quasi-equilibrium view is an adequate conceptual simplification.

We further analyzed the basic instability mechanisms of this model. We identified a moisture-stratiform instability, illustrated in Figure 11, which arises from the effect of mid-tropospheric humidity deficit on the depth of convection. We found that the net moistening effect of the second mode convective heating and the finite time to approach QE both act to reduce the growth rates, preferentially at low and high wavenumbers, respectively. These damping mechanisms help to select wavelengths of a few thousand kilometers as the fastest growing. An earlier study (KM06) included moisture in their convective parameterization, but their treatment is conceptually complicated and their analysis did not reveal a clear instability mechanism involving moisture. KM06 concluded that second baroclinic mode low-level moisture convergence plays a major role in the generation of the basic instability, whereas here this effect (included in $\mathrm{m}_{2}$ ) is found to damp the waves as discussed in section 3.b.2.

When the background convective heating profile is stronger in the upper troposphere than in the lower troposphere, the model contains an additional instability mechanism. This is named the direct-stratiform instability and is identified to be the same as the stratiform instability of M00. The direct stratiform instability mechanism, however, is inconsistent 
with the importance of moisture advection and the substantial tilt in the convective heating structure (especially at low wavenumbers) seen in the CSRM simulations of K07. The moisture-stratiform instability on the other hand is consistent with the CSRM simulation results, and is suggested as the main instability mechanism in these simulations, and likely in the real atmosphere under TOGA-COARE conditions as well. Whether and how this might change with the background mean state is a subject of further research. 


\section{Acknowledgements}

The author thanks three anonymous reviewers for their helpful comments and suggestions, and Brian Mapes, Masahiro Sugiyama, Chris Walker, Joe Andersen, an anonymous reviewer, and in particular, Dave Raymond for their helpful comments and suggestions on an earlier version of this paper. This work was supported partly by the Modeling, Analysis and Prediction (MAP) Program in the NASA Earth Science Division. 


\section{Appendices}

\section{A. Parameter Estimation}

In this appendix, we try to obtain rough estimates of the parameters used in the simple model. The CSRM simulations of K07 will be used to guide the estimates. In those simulations, a CSRM is coupled to linear gravity wave dynamics and convectively coupled waves spontaneously develop. The reader is referred to K07 for details about the simulations. We will use the first 20 days (i.e. the initial growth period) of the case with a lid at $14 \mathrm{~km}$, constant surface fluxes and a wavelength of $10,000 \mathrm{~km}$. A longer wavelength (and wave period) is preferable because convection and the large-scale wave can be expected to be closer to statistical equilibrium and the effect of the finite response time of convection, which would complicate the interpretation, is smaller.

We first construct CSRM counterparts of the simple model variables. Vertical mode decomposition (with a lid at $14 \mathrm{~km}$ ) is used to obtain $\mathrm{J}_{1}, \mathrm{~J}_{2}, \mathrm{w}_{1}, \mathrm{w}_{2}, \mathrm{~T}_{1}$, and $\mathrm{T}_{2}$. To be consistent with Eq. (4), the modes are normalized so that their absolute values average to 1. The subcloud layer is defined to be between the surface and $930 \mathrm{hPa}$. The variable $\mathrm{q}_{\text {mid }}$ is chosen to be that averaged over $400-600 \mathrm{hPa}$. It is important to emphasize that the simple model is a gross simplification of the CSRM simulations (and the real atmosphere). Therefore, viewing the CSRM results in the simple model framework is approximate and uncertainties in the resulting estimates are far greater than those implied by the goodness of the fit. We only intend to use these estimates as educated guesses of plausible values. 
With the above cautionary words in mind, we show in Figure 15a the regression of convective moistening $\left(Q_{2}\right)$ of $q_{\text {mid }}$ against the two convective heating modes $J_{1}$ and $J_{2}$. In the CSRM simulations, the large-scale advective tendencies are explicitly calculated and convective tendencies such as convective moistening and heating are computed as residuals, similar to e.g. Yanai et al. (1973). Contributions from $J_{1}$ and $J_{2}$ to convective moistening are shown in red and green, respectively. Their sum reproduces $\mathrm{Q}_{2}$ almost perfectly on the scale plotted and is omitted. Convective drying (negative $\mathrm{Q}_{2}$ ) is dominated by the first heating mode $\left(\mathrm{d}_{1}=1.3\right)$. The second heating mode has a moistening effect $\left(\mathrm{d}_{2}=-1.1\right)$. This represents a moistening (drying) effect in the mid-troposphere by congestus (stratiform) convection. Figure $15 \mathrm{~b}$ shows the regression of the $\mathrm{q}_{\mathrm{mid}}$ tendency due to vertical advection against $\mathrm{w}_{1}$ and $\mathrm{w}_{2}$, which yields $\mathrm{a}_{1}=1.6$ and $\mathrm{a}_{2}=0.0$. The effect of the second mode is small because the midtroposphere is around the nodal point of $\mathrm{w}_{2}$. Similar results are obtained from applying the vertical structures of $\mathrm{w}_{1}$ and $\mathrm{w}_{2}$ on the background moisture stratification and integrating from $400-600 \mathrm{hPa}$. The actual time derivative of $q_{\text {mid }}$ is the sum of convective drying and vertical moisture advection, and is substantially smaller because these two effects tend to cancel. In particular, there is a large compensation between adiabatic cooling and convective heating associated with the first mode: $\mathrm{w}_{1}$ is well correlated with $\mathrm{J}_{1}$ (correlation 0.98 ) and is only slightly larger (by $\sim 3 \%)$ than $\mathrm{J}_{1}$. Note that a larger adiabatic cooling $\left(\mathrm{w}_{1}\right)$ than convective heating $\mathrm{J}_{1}$ is consistent with the notion of a positive gross moist stability for the deep convective heating mode (e.g. Emanuel et al., 1994). Combined with the smallness of $a_{2}$, it appears reasonable to neglect moisture advection by $\mathrm{w}_{2}$ and combine convective drying and vertical moisture advection effects of the first mode, as in Eq. (7). This simplified 
treatment reproduces the total $\mathrm{q}_{\text {mid }}$ tendencies reasonably well (Figure 15c). While the estimates are subject to many uncertainties, that $m_{1}=a_{1}-d_{1}$ is positive, i.e. the net effect of deep convective heating, $\mathrm{J}_{1}$, is to make the mid-troposphere more humid, is a robust result.

We compute $\partial \mathrm{h}_{\mathrm{b}} / \partial \mathrm{t}$ as the mass weighted averages of moist static energy tendencies over the depth of the sub-cloud layer. Figure $15 \mathrm{~d}$ shows a regression of $\partial \mathrm{h}_{\mathrm{b}} / \partial \mathrm{t}$ by convection against $\mathrm{J}_{1}$ and $\mathrm{J}_{2}$ (Eq. (5) with $\mathrm{E}=0$ ). Contributions from large-scale vertical advection in the subcloud layer are smaller and neglected. This yields $b_{1}=1.0$ and $b_{2}=2.3$. A positive $b_{2}$ implies that convective heating in the lower troposphere is more effective at reducing $h_{b}$ than that in the upper troposphere on a per unit heating basis. This is perhaps somewhat counter-intuitive, but is quite clear in the CSRM simulations because boundary layer cooling and drying peaks before the maximum first mode heating, i.e. it is shifted towards the congestus phase (Figure 15d). Some indication of this is also seen in the 2day wave study of Haertel and Kiladis (2004).

We now try to constrain the parameters in Eq.(10). This formulation on the control of the height of convection is very approximate, so uncertainties in the estimates are large. To reflect these uncertainties, we simply choose $r_{0}=1$ and $r_{q}=0.7$. Figure 15 e indicates this is a plausible choice. A better fit can be achieved through a regression, but would convey a false sense of accuracy and is deemed more misleading than informative.

In Figure 15f, we plot the regression of subcloud layer moist static energy to the temperature averaged over the lower troposphere (between $930 \mathrm{hPa}$ and $500 \mathrm{hPa}$ ), assuming that the wave period is sufficiently long so that strict (or close to strict) 
statistical equilibrium is satisfied (Eq. (13)). This gives $\mathrm{F} \approx 4$. This is larger than $\partial \mathrm{s}^{*} / \partial \mathrm{T}$ at, say, $3 \mathrm{~km}(\sim 285 \mathrm{~K}, \sim 700 \mathrm{hPa})$, which is $\sim 3$. A possible reason for this is that factors such as entrainment may have diluted the $h_{b}$ variations as air parcels rise through the lower troposphere.

B. Connections between Eq.(3) and Eq.(19)

Let us first add a Newtonian cooling $\varepsilon$ in the thermodynamic equations in Eq. (3). Assuming a wave solution of the form $\exp (i \omega t-i k x)$, and diagonalizing the system, we have:

$$
\begin{aligned}
& \left(i \omega+\varepsilon+i k c_{j}\right)\left(-i w_{j}+k c_{j} T_{j}\right)=k c_{j} J_{j} \\
& \left(i \omega+\varepsilon-i k c_{j}\right)\left(i w_{j}+k c_{j} T_{j}\right)=k c_{j} J_{j}
\end{aligned}
$$

Eliminating $\mathrm{w}_{\mathrm{j}}$ leads to

$$
\left(i \omega+\varepsilon-i k c_{j}\right) T_{j}=J_{j} \frac{i \omega+\varepsilon}{i \omega+\varepsilon+i k c_{j}}
$$

For waves with periods substantially shorter than $2 \pi / \varepsilon$ ( $\sim 60$ days with our choice of $\varepsilon$ ), we have approximately

$$
\frac{\partial T_{j}}{\partial t}+c_{j} \frac{\partial T_{j}}{\partial x}=\frac{\omega}{\omega+k c_{j}} J_{j}-\varepsilon T_{j}
$$

Therefore, Eq. (3) is connected to Eq. (19) with

$$
J_{j}^{E q 3}=J_{j}^{E q 19}\left(1+\frac{k c_{j}}{\omega}\right)
$$

Eq. (39) indicates that convective heating is more effective in forcing temperature variations in the one-way wave equations. This is particularly true for the first vertical mode. For waves with a phase speed of $25 \mathrm{~m} / \mathrm{s}$, the first vertical mode heating is three 
times as effective and the second vertical mode heating is twice as effective in the oneway wave equations compared to the two-way wave equations.

C. Limiting cases with general parameter choices

In this appendix, we consider the limiting cases with general parameter choices to extend the results with the simplifying parameter choices presented in Sections $3 \mathrm{~b}$ and $3 \mathrm{c}$. We shall consider the system to be in SQE. The effect of $\tau_{\mathrm{J}}$ is similar to that with the simplifying parameter choices.

When $\mathrm{f}=1$, Eq.(23) becomes

$$
J_{1, e q}=\frac{1}{b_{1}+b_{2} \gamma_{0}}\left(b_{2} \gamma_{q} q-F \frac{\partial T_{1}}{\partial t}\right)
$$

We have continued to neglect $\mathrm{T}_{1}$ 's contribution in $\mathrm{q}^{+}$. At SQE, we have

$$
\begin{aligned}
& \frac{\partial T_{1}}{\partial t}+\hat{c}_{1} \frac{\partial T_{1}}{\partial x}=K_{1} \gamma_{q} q-\hat{\varepsilon}_{1} T_{1} \\
& \frac{\partial q}{\partial t}=-\hat{m}_{1} \frac{F}{b_{1}} \frac{\partial T_{1}}{\partial t}-\hat{m}_{2} \gamma_{q} q
\end{aligned}
$$

where

$$
\begin{aligned}
& \left(\hat{\varepsilon}_{1}, \hat{c}_{1}\right) \equiv\left(\varepsilon, c_{1}\right) \frac{b_{1}+b_{2} \gamma_{0}}{F+b_{1}+b_{2} \gamma_{0}} \\
& \hat{m}_{1} \equiv \frac{m_{1}+m_{2} \gamma_{0}}{1+b_{2} \gamma_{0} / b_{1}} \\
& \hat{m}_{2} \equiv \frac{b_{1} m_{2}-b_{2} m_{1}}{b_{1}+b_{2} \gamma_{0}} \\
& K_{1} \equiv \frac{b_{2}}{F+b_{1}+b_{2} \gamma_{0}}
\end{aligned}
$$


Here $\mathrm{T}_{2}$ is forced by modes of Eq. (41) and does not feed back onto $\mathrm{T}_{1}$ and $\mathrm{q}$. This is the same as the limiting case I discussed in section 3.b.1 except with the parameters modified and an additional moisture equation.

When $\mathrm{f}=0$, we have

$$
J_{1, e q}=\frac{1}{b_{1}+b_{2} \gamma_{0}}\left(b_{2} \gamma_{q} q-F \frac{\partial T_{2}}{\partial t}\right)
$$

Therefore:

$$
\begin{aligned}
& \frac{\partial T_{2}}{\partial t}+\hat{c}_{2} \frac{\partial T_{2}}{\partial x}=K_{2} \gamma_{q} q-\hat{\varepsilon}_{2} T_{2} \\
& \frac{\partial q}{\partial t}=-\hat{m}_{1} \frac{F}{b_{1}} \frac{\partial T_{2}}{\partial t}-\hat{m}_{2} \gamma_{q} q
\end{aligned}
$$

where

$$
\begin{aligned}
& \left(\hat{\varepsilon}_{2}, \hat{c}_{2}\right) \equiv\left(\varepsilon, c_{2}\right) \frac{b_{1}+b_{2} \gamma_{0}}{b_{1}+\left(b_{2}+F\right) \gamma_{0}} \\
& K_{2} \equiv \frac{-b_{1}}{b_{1}+\left(b_{2}+F\right) \gamma_{0}}
\end{aligned}
$$

Here $T_{1}$ is forced by the modes of Eq. (44), and does not feedback on $T_{2}$ and q. This is the same as the limiting case II discussed in section 3.b.2 except with the modified parameters. Comparing Eq. (44) and Eq. (41), we see that the two systems have the same form. In terms of linear stability, the main difference is in the coefficients in front of $q$ in the temperature equation, $\mathrm{K}_{1}$ and $\mathrm{K}_{2}$. Using our normative parameter values and allowing for a reasonable range, $K_{1}$ is close to 0.4 , and $K_{2}$ is close to -1 . Therefore, the $f=1$ case with general parameter choices may be viewed as having a negative effective $\gamma_{\mathrm{q}}$. 


\section{References}

Arakawa, A. and W. H. Schubert, 1974: Interaction of a Cumulus Cloud Ensemble with Large-Scale Environment.1. Journal of the Atmospheric Sciences, 31, 674-701.

Brown, R. G. and C. D. Zhang, 1997: Variability of midtropospheric moisture and its effect on cloud-top height distribution during TOGA COARE. Journal of the Atmospheric Sciences, 54, 2760-2774.

Derbyshire, S. H., I. Beau, P. Bechtold, J. Y. Grandpeix, J. M. Piriou, J. L. Redelsperger, and P. M. M. Soares, 2004: Sensitivity of moist convection to environmental humidity. Quarterly Journal Of The Royal Meteorological Society, 130, 3055-3079.

Emanuel, K., 1993: The effect of convective response time on WISHE modes. Journal of the Atmospheric Sciences, 50, 1763-1775.

Emanuel, K. A., 1986: An Air Sea Interaction Theory For Tropical Cyclones.1. SteadyState Maintenance. Journal Of The Atmospheric Sciences, 43, 585-604.

_- 1987: An Air-Sea Interaction-Model Of Intraseasonal Oscillations In The Tropics. Journal Of The Atmospheric Sciences, 44, 2324-2340.

__ 1995: The Behavior Of A Simple Hurricane Model Using A Convective Scheme Based On Subcloud-Layer Entropy Equilibrium. Journal Of The Atmospheric Sciences, $\mathbf{5 2 , 3 9 6 0 - 3 9 6 8 . ~}$

Emanuel, K. A., J. D. Neelin, and C. S. Bretherton, 1994: On Large-Scale Circulations in Convecting Atmospheres. Quarterly Journal of the Royal Meteorological Society, 120, $1111-1143$ 
Haertel, P. T. and G. N. Kiladis, 2004: Dynamics of 2-day equatorial waves. Journal of the Atmospheric Sciences, 61, 2707-2721.

Khairoutdinov, M. and D. Randall, 2006: High-resolution simulation of shallow-to-deep convection transition over land. Journal of the Atmospheric Sciences, in press.

Khouider, B. and A. J. Majda, 2006a: A simple multicloud parameterization for convectively coupled tropical waves. Part I: Linear analysis. Journal of the Atmospheric Sciences, 63, 1308-3123.

- , 2006b: Multicloud convective parametrizations with crude vertical structure. Theoretical And Computational Fluid Dynamics, 20, 351-375.

Kuang, Z., 2007: Modeling the interaction between cumulus convection and linear waves in a limited domain cloud system resolving model. Journal of the Atmospheric Sciences, submitted.

Kuang, Z. M. and C. Bretherton, 2006: A mass flux scheme view of a high-resolution simulation of a transition from shallow to deep cumulus convection. Journal of the Atmospheric Sciences, 63, 1895-1909.

Lindzen, R. S., 1974: Wave-CISK in the tropics. Journal of the Atmospheric Sciences, 32, 156-179.

Majda, A. J. and M. G. Shefter, 2001: Models for stratiform instability and convectively coupled waves. Journal of the Atmospheric Sciences, 58, 1567-1584.

Majda, A. J., B. Khouider, G. Kiladis, K. H. Straub, and M. G. Shefter, 2004: A model for convectively coupled tropical waves: Nonlinearity, rotation, and comparison with observations. Journal of the Atmospheric Sciences, 61, 2188-2205. 
Mapes, B., S. Tulich, J. Lin, and P. Zuidema, 2006: The mesoscale convection life cycle: Building block or prototype for large-scale tropical waves? Dynamics Of Atmospheres And Oceans, 42, 3-29.

Mapes, B. E., 2000: Convective inhibition, subgrid-scale triggering energy, and stratiform instability in a toy tropical wave model. Journal of the Atmospheric Sciences, $\mathbf{5 7}, 1515-1535$.

Neelin, J. D. and J. Y. Yu, 1994: Modes Of Tropical Variability Under Convective Adjustment And The Madden-Julian Oscillation.1. Analytical Theory. Journal Of The Atmospheric Sciences, 51, 1876-1894.

Neelin, J. D., I. M. Held, and K. H. Cook, 1987: Evaporation-Wind Feedback And LowFrequency Variability In The Tropical Atmosphere. Journal Of The Atmospheric Sciences, 44, 2341-2348.

Parsons, D. B., K. Yoneyama, and J. L. Redelsperger, 2000: The evolution of the tropical western Pacific atmosphere-ocean system following the arrival of a dry intrusion. Quarterly Journal Of The Royal Meteorological Society, 126, 517-548.

Raymond, D. J., 1995: Regulation of Moist Convection over the West Pacific Warm Pool. Journal of the Atmospheric Sciences, 52, 3945-3959.

— 2000: Thermodynamic control of tropical rainfall. Quarterly Journal of the Royal Meteorological Society, 126, 889-898.

Redelsperger, J. L., D. B. Parsons, and F. Guichard, 2002: Recovery processes and factors limiting cloud-top height following the arrival of a dry intrusion observed during TOGA COARE. Journal Of The Atmospheric Sciences, 59, 2438-2457. 
Ridout, J. A., 2002: Sensitivity of tropical Pacific convection to dry layers at mid- to upper levels: Simulation and parameterization tests. Journal Of The Atmospheric Sciences, 59, 3362-3381.

Roca, R., J. P. Lafore, C. Piriou, and J. L. Redelsperger, 2005: Extratropical dry-air intrusions into the West African monsoon midtroposphere: An important factor for the convective activity over the Sahel. Journal Of The Atmospheric Sciences, 62, 390-407.

Sherwood, S. C., 1999: Convective precursors and predictability in the tropical western Pacific. Monthly Weather Review, 127, 2977-2991.

Straub, K. H. and G. N. Kiladis, 2002: Observations of a convectively coupled Kelvin wave in the eastern Pacific ITCZ. Journal of the Atmospheric Sciences, 59, 30-53.

Takemi, T., O. Hirayama, and C. H. Liu, 2004: Factors responsible for the vertical development of tropical oceanic cumulus convection. Geophysical Research Letters, 31.

Tulich, S., D. Randall, and B. Mapes, 2006: Vertical-Mode and Cloud Decomposition of Large-Scale Convectively Coupled Gravity Waves in a Two-Dimensional CloudResolving Model. Journal of the Atmospheric Sciences.

Wang, B., 1988: Dynamics Of Tropical Low-Frequency Waves - An Analysis Of The Moist Kelvin Wave. Journal Of The Atmospheric Sciences, 45, 2051-2065.

Webster, P. J. and R. Lukas, 1992: Toga Coare - the Coupled Ocean Atmosphere Response Experiment. Bulletin of the American Meteorological Society, 73, 1377-1416.

Wheeler, M., G. N. Kiladis, and P. J. Webster, 2000: Large-scale dynamical fields associated with convectively coupled equatorial waves. Journal of the Atmospheric Sciences, 57, 613-640. 
Yanai, M., S. Esbensen, and J. H. Chu, 1973: Determination Of Bulk Properties Of Tropical Cloud Clusters From Large-Scale Heat And Moisture Budgets. Journal Of The Atmospheric Sciences, 30, 611-627.

Yu, J. Y. and J. D. Neelin, 1994: Modes Of Tropical Variability Under Convective Adjustment And The Madden-Julian Oscillation.2. Numerical Results. Journal Of The Atmospheric Sciences, 51, 1895-1914. 


\section{Figure Captions}

Figure 1 Phase speed (left) and growth rate (right) as functions of wavenumber from the linearized version of the full model described in section 2c (with 2-way wave equations), using normative parameter values. Modes with positive growth rates are highlighted with circles in the phase speed diagram. The phase speeds are symmetric about 0 .

Figure 2 Physical patterns of the eigenmodes of the linearized full model described in section 2c (with 2-way wave equations) for an eastward propagating wave with a wavelength of $8640 \mathrm{~km}$. Normative values are used for all parameters. (a) T1 (solid), T2 (dashed) and q (dotted) as functions of x (zonal distance). (b) J1 (solid) and J2 (dashed) as functions of $\mathrm{x}$. (c) Zonal and height pattern of the combined temperature anomaly. The contour interval is $0.5 \mathrm{~K}$. (d) Zonal and height pattern of the combined convective heating anomaly. The contour interval is $2 \mathrm{~K} / \mathrm{day}$. In both (c) and (d), negative contours are dashed and the zero contour is omitted.

Figure 3 Phase lag between $\mathrm{J} 1$ and $-\mathrm{J} 2$ as a function of wavenumber for the linearized full model described in section $2 \mathrm{c}$ with normative parameter values.

Figure 4 Maximum linear growth rates for the linearized full model described in section 2c with individual parameters varied and the other parameters kept at their normative values.

Figure 5 Same as Figure 1 except for the simplified version described in section 3a.

Figure 6 Same as Figure 2 except for the simplified version described in section 3a and a contour interval of $0.5 \mathrm{~K} /$ day in $(\mathrm{d})$. 
Figure 7 Same as Figure 4 but for the system described in section 3a.

Figure 8 Same as Figure 6 except for the limiting case I ( $\mathrm{f}=1)$ described in section 3.b.1 with $\mathrm{b} 2=0, \gamma 0=0, \varepsilon=0, \tau \mathrm{J}=0, \mathrm{~m} 2=0$.

Figure 9 Same as Figure 8, except for limiting case II ( $\mathrm{f}=0)$ described in section 3.b.2 with $\mathrm{b} 2=0, \gamma 0=0, \varepsilon=0, \tau \mathrm{J}=0, \mathrm{~m} 2=0$.

Figure 10 Growth rates as a function of wavenumber for limiting case II described in section $3 . b .2$ with $\mathrm{m} 2=\tau \mathrm{J}=\varepsilon=0$ (thick solid), $\tau \mathrm{J}=\varepsilon=0$ (thin solid), $\mathrm{m} 2=\varepsilon=0$ (dotted), and $\varepsilon=0$ (diamond symbols), and none of $\mathrm{m} 2, \tau \mathrm{J}, \varepsilon$ is zero (circles). When a parameter is not zero, it takes its normative value.

Figure 11 A schematic of the moisture-stratiform instability, illustrated for an eastward propagating wave viewed in a reference frame that follows the wave. All fields shown are anomalies. We start with (a) temperature and vertical velocity (arrows) anomalies associated with the wave. The large-scale lifting cools the lower troposphere as part of the wave signal. This induces a positive deep convection anomaly, which cools the subcloud layer to maintain quasi-equilibrium with the large-scale flow (b). The deep convection anomaly also makes the midtroposphere more humid (c). An anomalously moist mid-troposphere allows convection to reach higher, while an anomalously dry one makes convection lower. This produces a convective heating anomaly pattern that is in phase with the original temperature anomaly and causes instability (d).

Figure 12 Growth rates as a function of wavenumber and $\mathrm{f}$ with $\mathrm{b} 2=0, \gamma 0=0, \varepsilon=0, \tau \mathrm{J}=0$, $\mathrm{m} 2=0$. 
Figure 13 (a) Linear growth rate of the unstable mode as a function of wavenumber and r0 for the system described by Eq. (32) and with $\mathrm{f}=0.5$. (b) Same as (a) but with the effect of a $2 \mathrm{hr}$ adjustment time to QE included.

Figure 14 Same as Figure 8, except for the system described by Eq. (32), and with $\mathrm{f}=0.5$ and $\gamma 0=-0.2$.

Figure 15 (a) Contributions to convective drying of the mid-troposphere ( $\partial \mathrm{qmid} / \partial \mathrm{t}) \operatorname{conv}$ (black) by -d1J1 (red) and -d2J2 (green) based on a linear regression. (b) Contributions to advective moistening of the mid-troposphere ( $\partial \mathrm{qmid} / \partial \mathrm{t}) \mathrm{adv}$ (black) by a1w1 (red) and a2w2 (green) based on a linear regression. (c) Total tendencies of the mid-troposphere humidity $\partial \mathrm{qmid} / \partial \mathrm{t}$ (black) and (a1-d1)J1-d2J2 (red). (d) A linear regression of convective tendencies of boundary moist static energy $\partial \mathrm{hb} / \partial \mathrm{t}$ (black) against J1 and J2. (e) The second mode heating (black) and $\mathrm{rq}(1.5 \mathrm{~T} 1$-qmid) (red) with $\mathrm{rq}=0.7$. (f) A linear regression of hb against lower tropospheric temperature Tlow. 

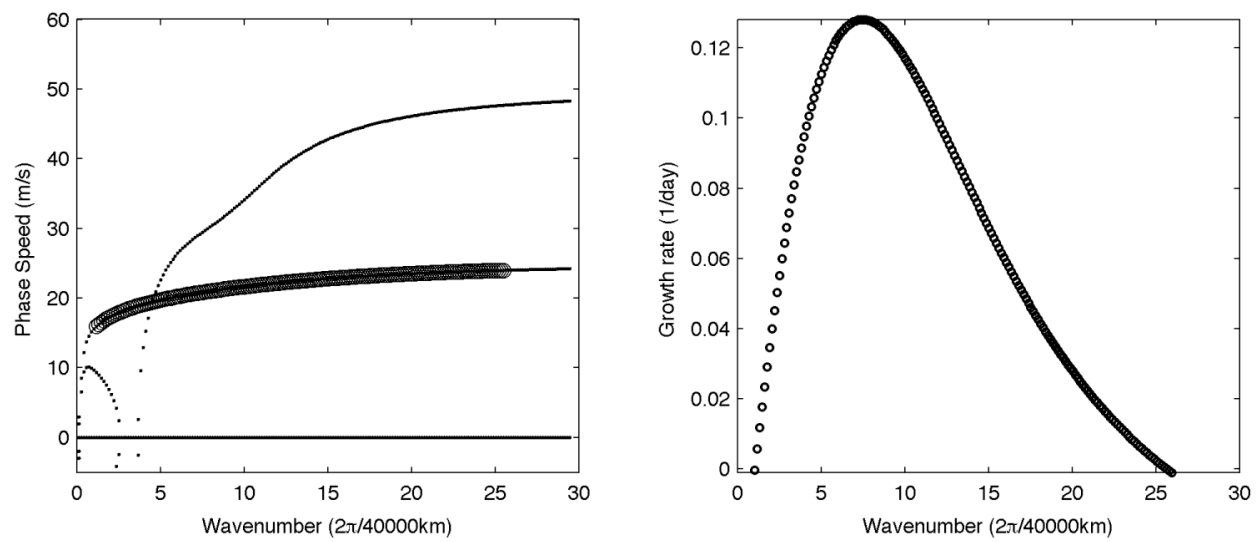

Figure 1 Phase speed (left) and growth rate (right) as functions of wavenumber from the linearized version of the full model described in section 2c (with 2-way wave equations), using normative parameter values. Modes with positive growth rates are highlighted with circles in the phase speed diagram. The phase speeds are symmetric about 0. 
(a)

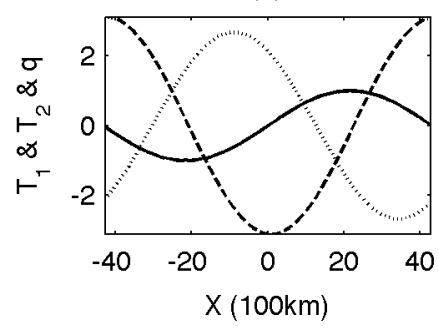

(c) $\mathrm{T}^{\prime}(0.5 \mathrm{~K})$

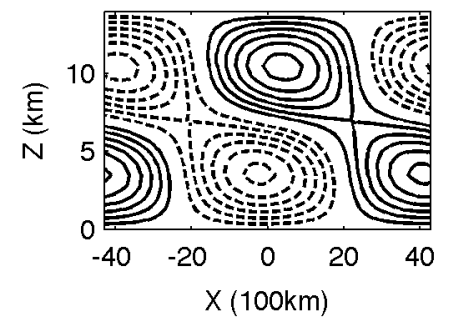

(b)

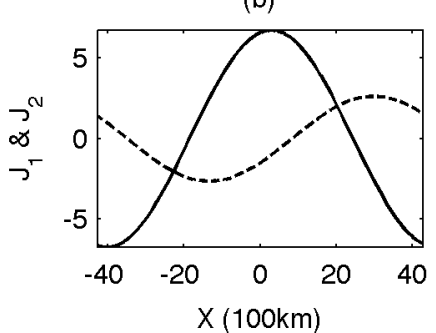

(d) J' (2K/day)

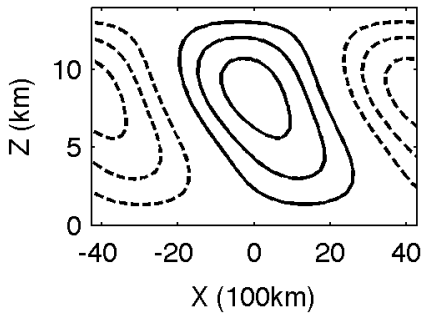

Figure 2 Physical patterns of the eigenmodes of the linearized full model described in section $2 \mathrm{c}$ (with 2-way wave equations) for an eastward propagating wave with a wavelength of $8640 \mathrm{~km}$. Normative values are used for all parameters. (a) $\mathrm{T}_{1}$ (solid), $\mathrm{T}_{2}$ (dashed) and $\mathrm{q}$ (dotted) as functions of $\mathrm{x}$ (zonal distance). (b) $\mathrm{J}_{1}$ (solid) and $\mathrm{J}_{2}$ (dashed) as functions of $x$. (c) Zonal and height pattern of the combined temperature anomaly. The contour interval is $0.5 \mathrm{~K}$. (d) Zonal and height pattern of the combined convective heating anomaly. The contour interval is $2 \mathrm{~K} /$ day. In both (c) and (d), negative contours are dashed and the zero contour is omitted. 


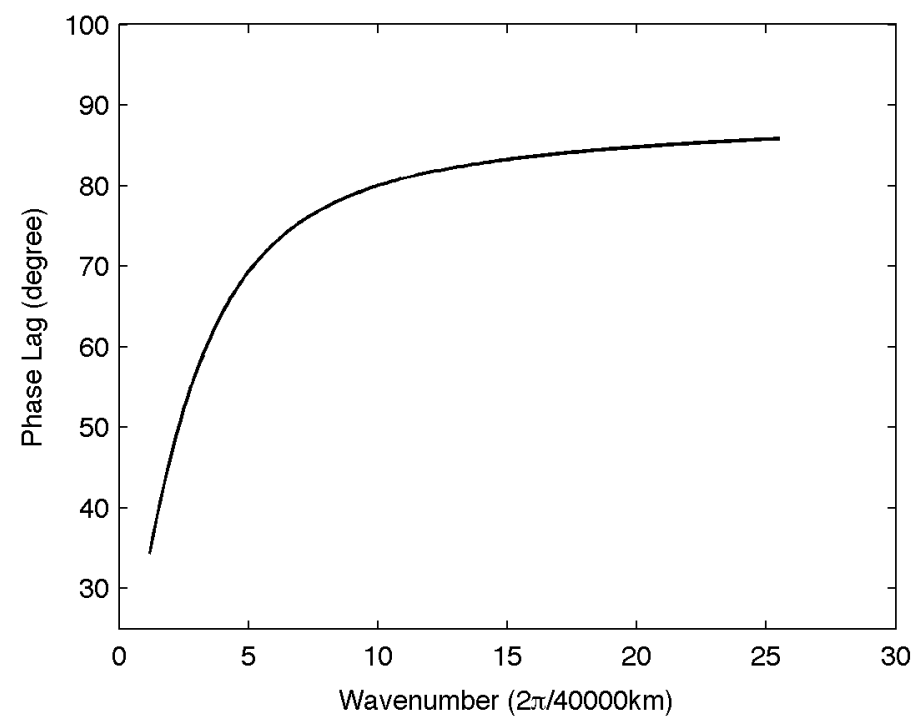

Figure 3 Phase lag between $\mathrm{J}_{1}$ and $-\mathrm{J}_{2}$ as a function of wavenumber for the linearized full model described in section $2 \mathrm{c}$ with normative parameter values. 

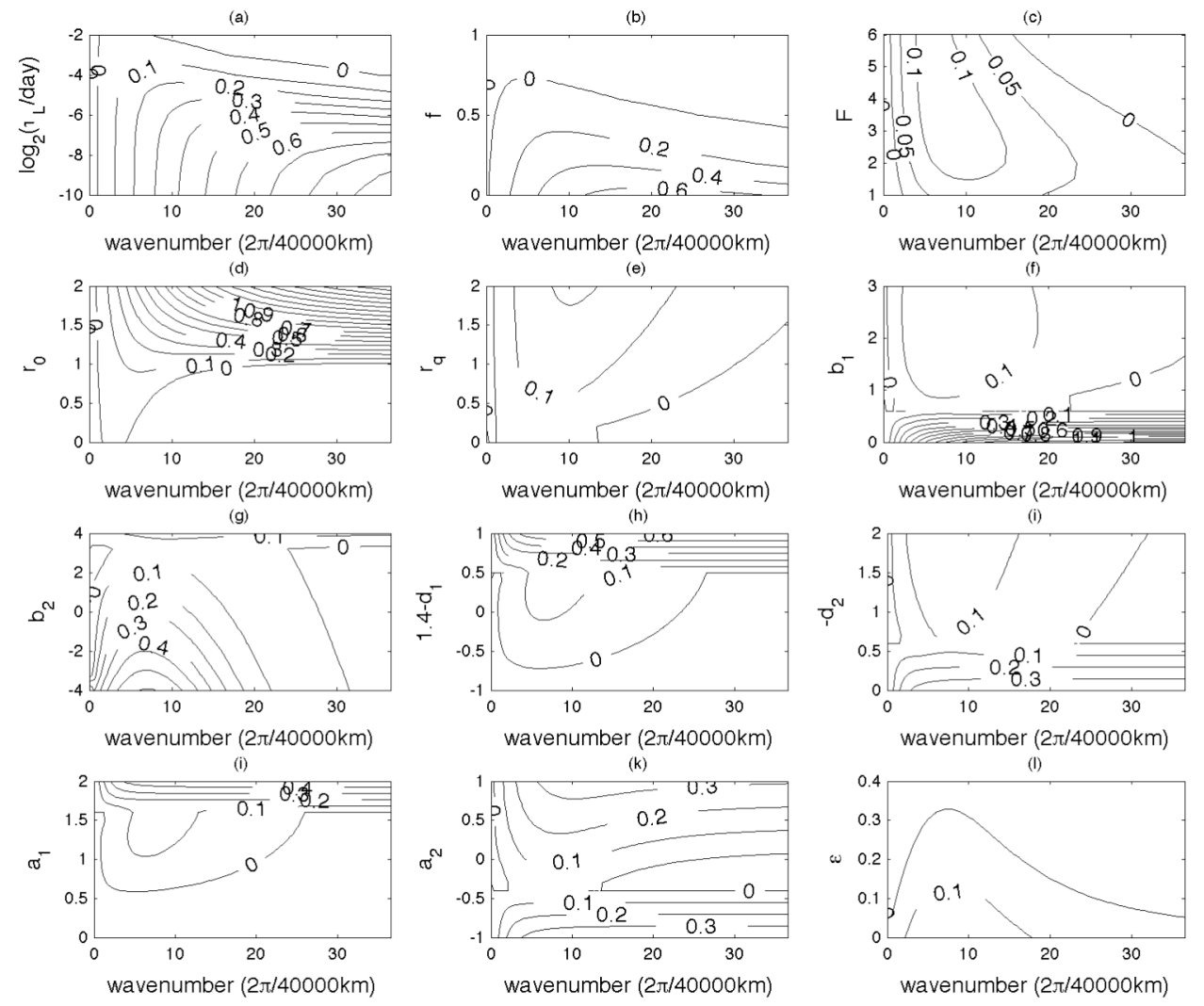

Figure 4 Maximum linear growth rates for the linearized full model described in section $2 \mathrm{c}$ with individual parameters varied and the other parameters kept at their normative values. 

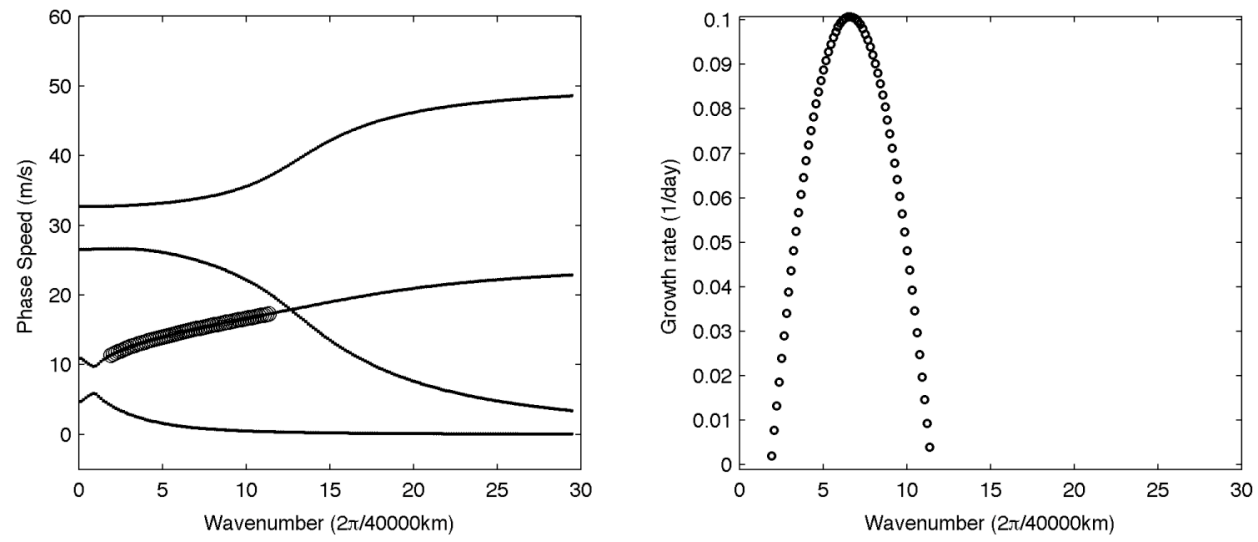

Figure 5 Same as Figure 1 except for the simplified version described in section 3a. 
(a)

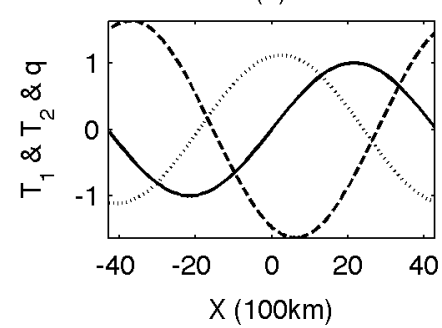

(c) $\mathrm{T}^{\prime}(0.5 \mathrm{~K})$

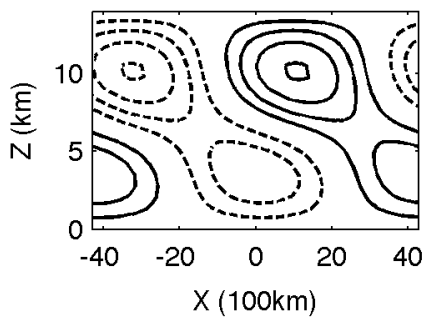

(b)

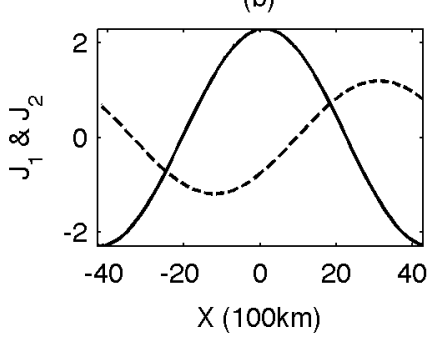

(d) J' (0.5K/day)

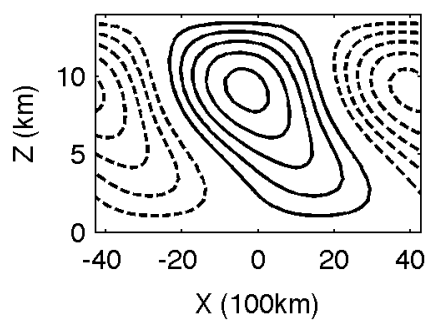

Figure 6 Same as Figure 2 except for the simplified version described in section 3a and a contour interval of $0.5 \mathrm{~K} /$ day in $(\mathrm{d})$. 

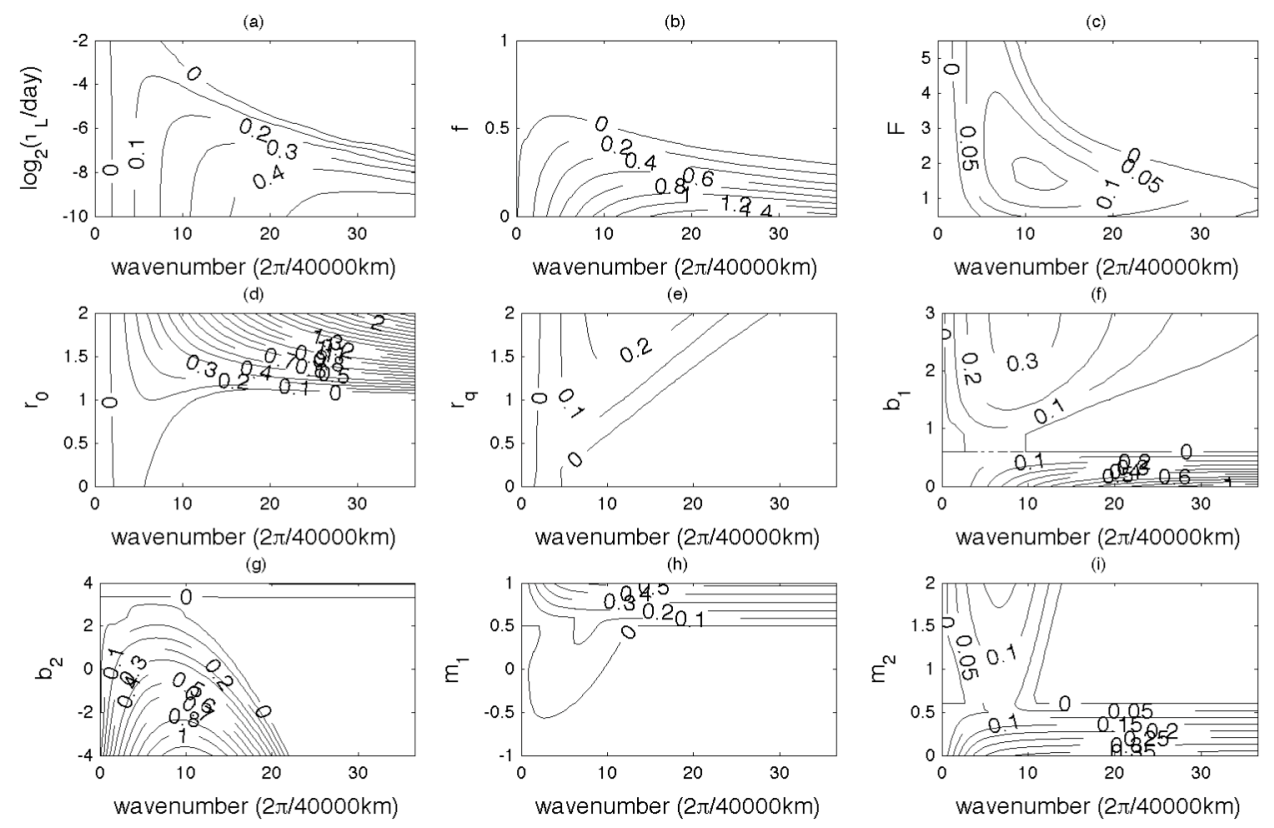

Figure 7 Same as Figure 4 but for the system described in section 3a. 
(a)

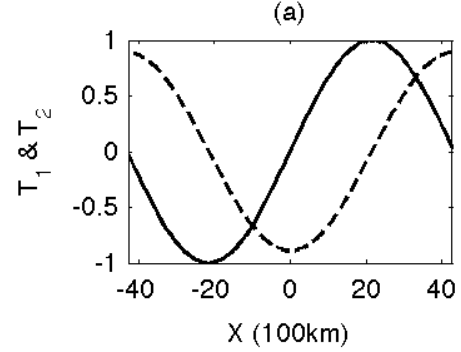

(c) $\mathrm{T}^{\prime}(0.5 \mathrm{~K})$

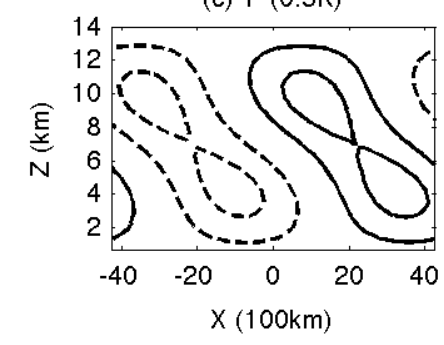

(b)

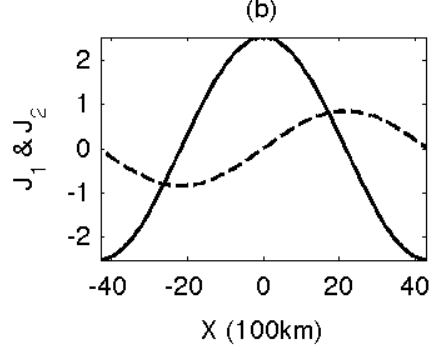

(d) J' $(0.5 \mathrm{~K} /$ day $)$

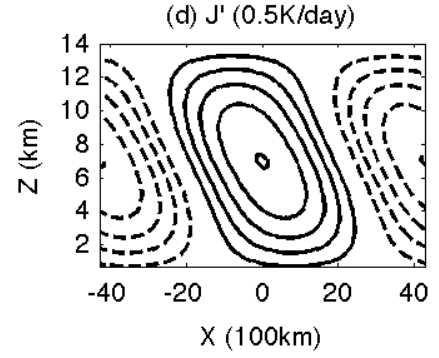

Figure 8 Same as Figure 6 except for the limiting case $\mathrm{I}(\mathrm{f}=1)$ described in section 3.b.1 with $\mathrm{b}_{2}=0, \gamma_{0}=0, \varepsilon=0, \tau_{\mathrm{J}}=0, \mathrm{~m}_{2}=0$. 

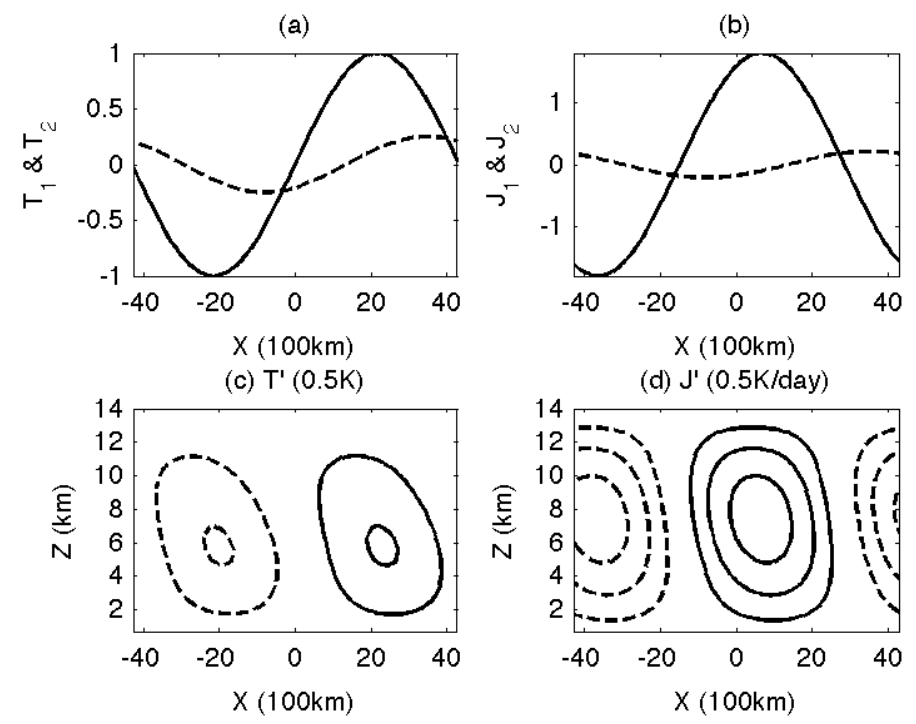

Figure 9 Same as Figure 8, except for limiting case II ( $\mathrm{f}=0$ ) described in section 3.b.2 with $\mathrm{b}_{2}=0, \gamma_{0}=0, \varepsilon=0, \tau_{\mathrm{J}}=0, \mathrm{~m}_{2}=0$. 


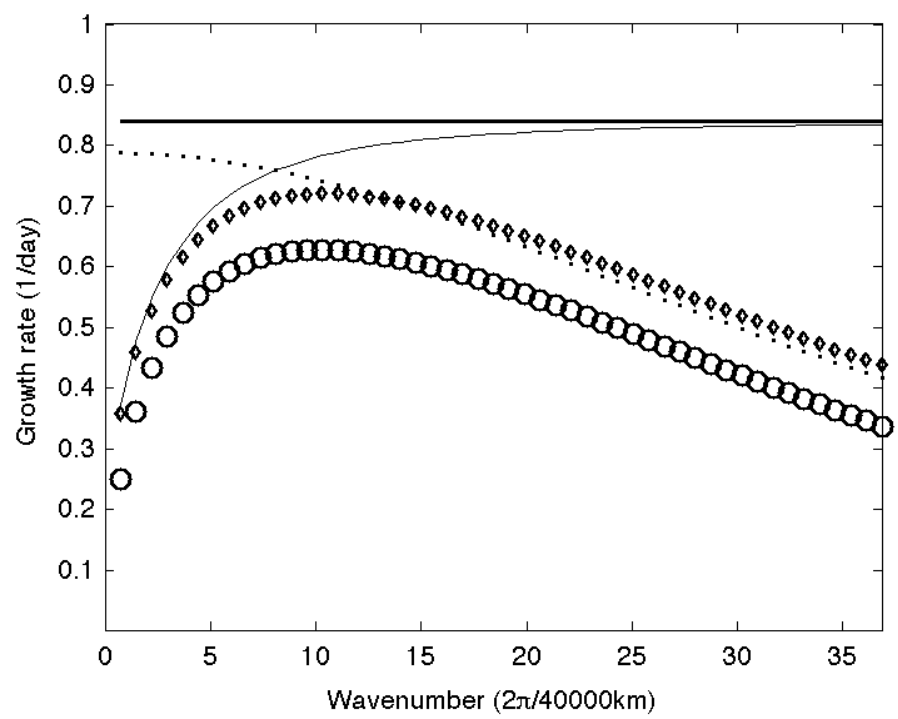

Figure 10 Growth rates as a function of wavenumber for limiting case II described in section 3.b. 2 with $\mathrm{m}_{2}=\tau_{\mathrm{J}}=\varepsilon=0$ (thick solid), $\tau_{\mathrm{J}}=\varepsilon=0$ (thin solid), $\mathrm{m}_{2}=\varepsilon=0$ (dotted), and $\varepsilon=0$ (diamond symbols), and none of $\mathrm{m}_{2}, \tau_{\mathrm{J}}, \varepsilon$ is zero (circles). When a parameter is not zero, it takes its normative value. 
(a)

An eastward propagating wave

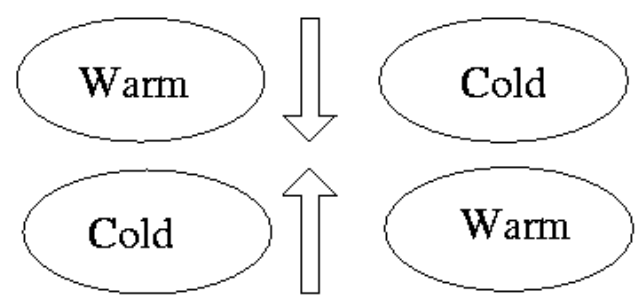

(b)

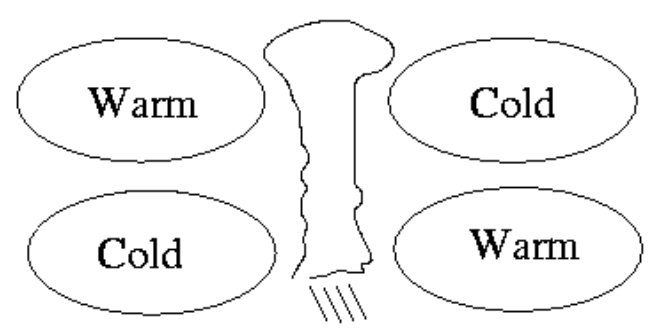

(c)

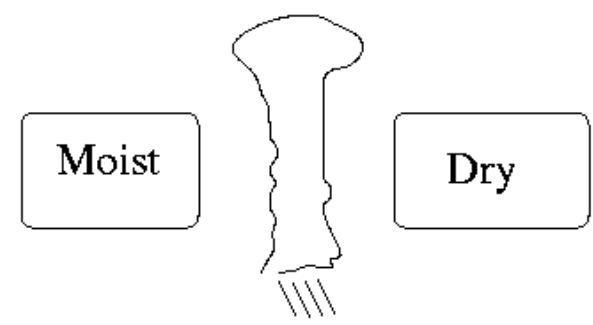

(d)
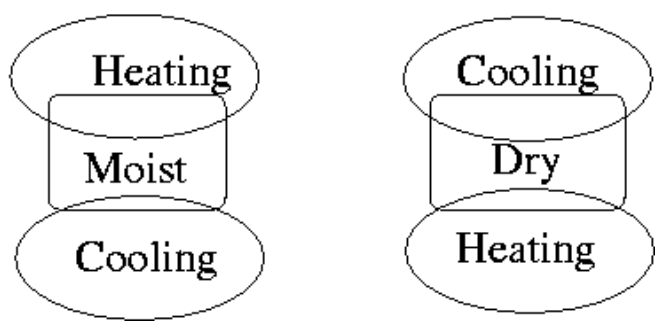

Figure 11 A schematic of the moisture-stratiform instability, illustrated for an eastward propagating wave viewed in a reference frame that follows the wave. All fields shown are anomalies. We start with (a) temperature and vertical velocity (arrows) anomalies associated with the wave. The large-scale lifting cools the lower troposphere as part of the wave signal. This induces a positive deep convection anomaly, which cools the subcloud layer to maintain quasi-equilibrium with the large-scale flow (b). The deep convection anomaly also makes the midtroposphere more humid (c). An anomalously moist mid-troposphere allows convection to reach higher, while an anomalously dry one makes convection lower. This produces a convective heating anomaly pattern that is in phase with the original temperature anomaly and causes instability $(\mathrm{d})$. 


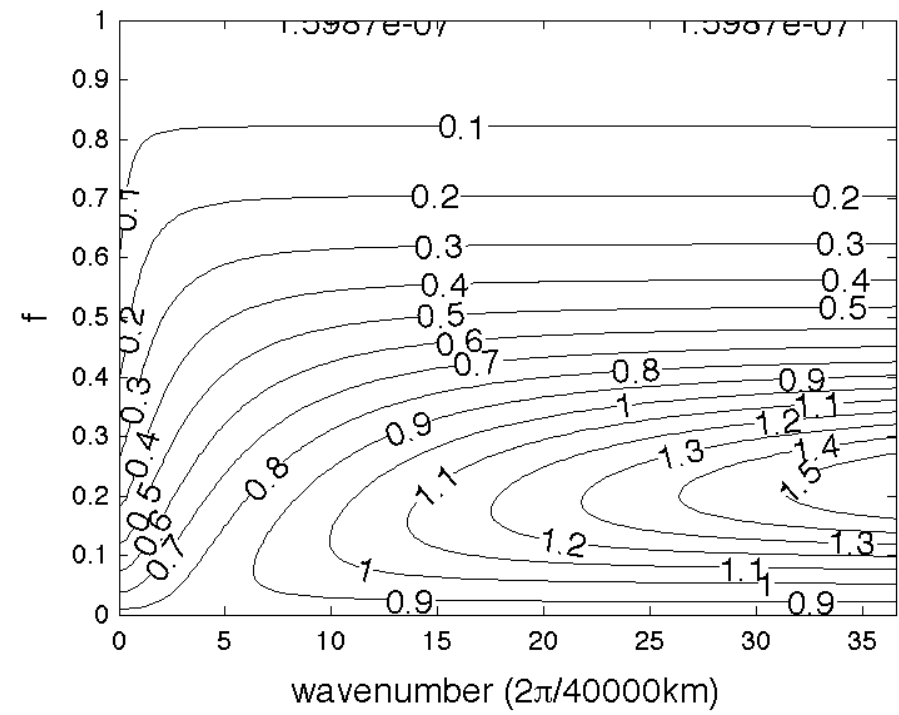

Figure 12 Growth rates as a function of wavenumber and $\mathrm{f}$ with $\mathrm{b}_{2}=0, \gamma_{0}=0, \varepsilon=0, \tau_{\mathrm{J}}=0$, $\mathrm{m}_{2}=0$. 

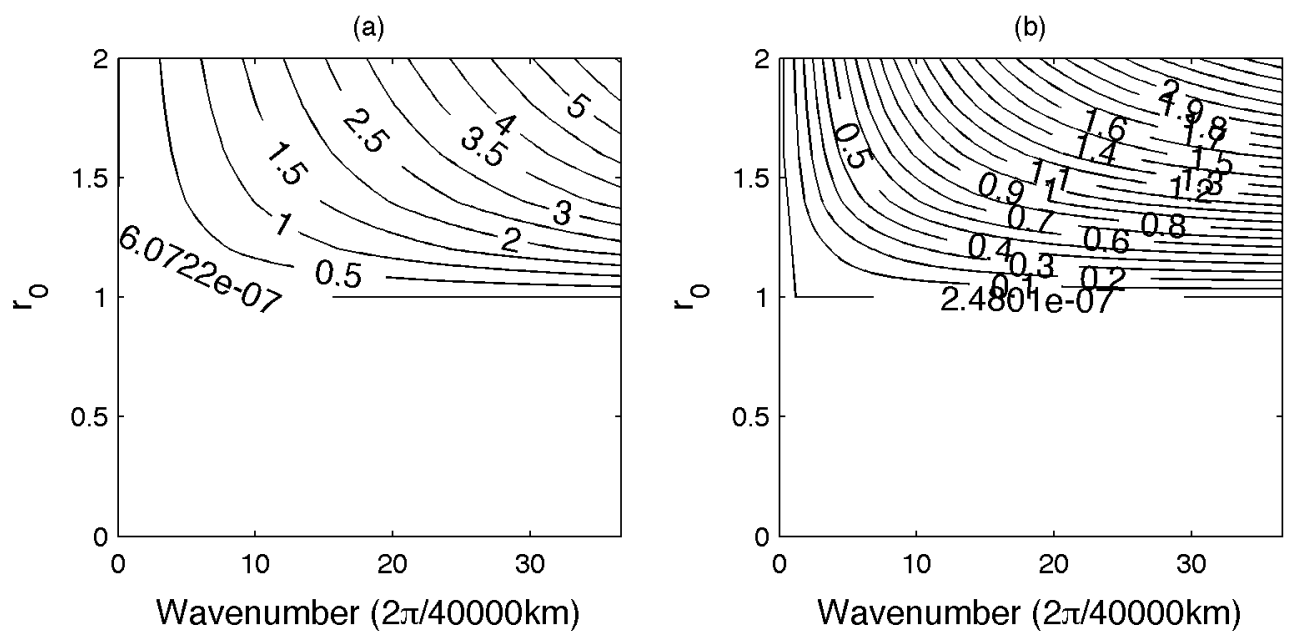

Figure 13 (a) Linear growth rate of the unstable mode as a function of wavenumber and $r_{0}$ for the system described by Eq. (32) and with $\mathrm{f}=0.5$. (b) Same as (a) but with the effect of a $2 \mathrm{hr}$ adjustment time to $\mathrm{QE}$ included. 
(a)

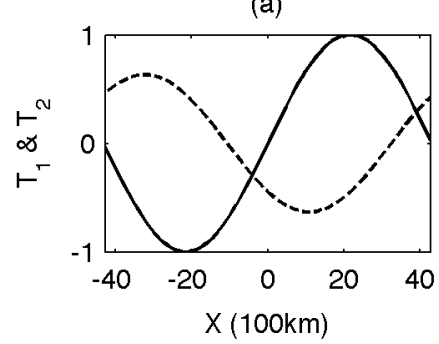

(c) $\mathrm{T}^{\prime}(0.5 \mathrm{~K})$

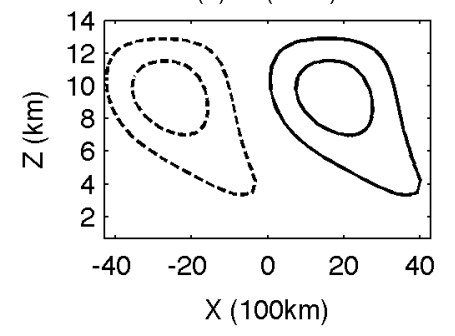

(b)

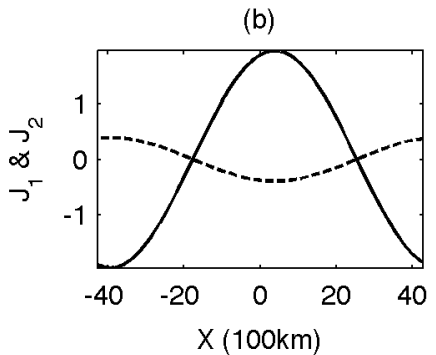

(d) J' $(0.5 \mathrm{~K} /$ day $)$

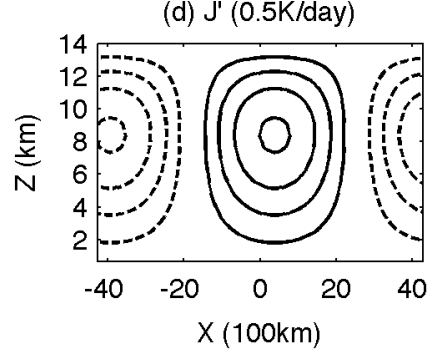

Figure 14 Same as Figure 8, except for the system described by Eq. (32), and with $\mathrm{f}=0.5$ and $\gamma_{0}=-0.2$. 


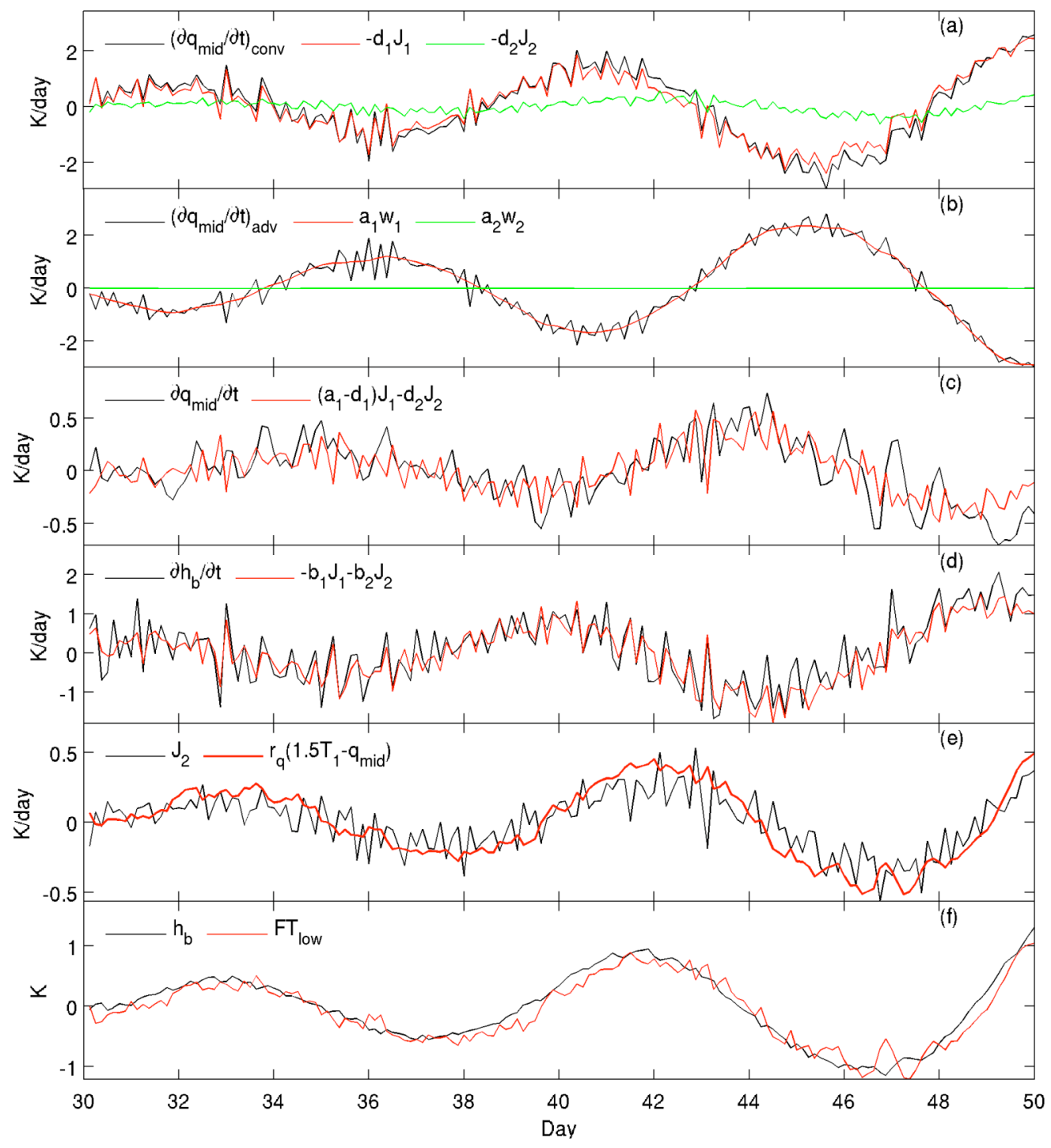

Figure 15 (a) Contributions to convective drying of the mid-troposphere $\left(\partial \mathrm{q}_{\text {mid }} / \partial \mathrm{t}\right)_{\text {conv }}$ (black) by $-\mathrm{d}_{1} \mathrm{~J}_{1}$ (red) and $-\mathrm{d}_{2} \mathrm{~J}_{2}$ (green) based on a linear regression. (b) Contributions to advective moistening of the mid-troposphere $\left(\partial \mathrm{q}_{\mathrm{mid}} / \partial \mathrm{t}\right)_{\mathrm{adv}}$ (black) by $\mathrm{a}_{1} \mathrm{w}_{1}(\mathrm{red})$ and $\mathrm{a}_{2} \mathrm{w}_{2}$ (green) based on a linear regression. (c) Total tendencies of the mid-troposphere humidity $\partial \mathrm{q}_{\text {mid }} / \partial \mathrm{t}$ (black) and $\left(\mathrm{a}_{1}-\mathrm{d}_{1}\right) \mathrm{J}_{1}-\mathrm{d}_{2} \mathrm{~J}_{2}$ (red). (d) A linear regression of convective tendencies of boundary moist static energy $\partial \mathrm{h}_{\mathrm{b}} / \partial \mathrm{t}$ (black) against $\mathrm{J}_{1}$ and $\mathrm{J}_{2}$. (e) The second mode heating (black) and $\mathrm{r}_{\mathrm{q}}\left(1.5 \mathrm{~T}_{1}-\mathrm{q}_{\mathrm{mid}}\right)(\mathrm{red})$ with $\mathrm{r}_{\mathrm{q}}=0.7$. (f) A linear regression of $\mathrm{h}_{\mathrm{b}}$ against lower tropospheric temperature $\mathrm{T}_{\text {low }}$. 


\section{Tables:}

Table 1 A summary of parameters used in the simple model

\begin{tabular}{|c|c|c|c|}
\hline Symbol & $\begin{array}{l}\text { Normative } \\
\text { values }\end{array}$ & Unit & Description \\
\hline $\mathrm{b}_{1}, \mathrm{~b}_{2}$ & 1,2 & - & Reduction in $h_{b}$ per unit $J_{1}$ and $J_{2}$ (Eq.(5)) \\
\hline $\mathrm{a}_{1}, \mathrm{a}_{2}$ & $1.4,0$ & - & Increase in $\mathrm{q}_{\text {mid }}$ (by advection) per unit $\mathrm{w}_{1}$ and $\mathrm{w}_{2}$, Eq. (6) \\
\hline $\mathrm{d}_{1}, \mathrm{~d}_{2}$ & $1.1,-1$ & - & Decrease in $\mathrm{q}_{\text {mid }}$ per unit $\mathrm{J}_{1}$ and $\mathrm{J}_{2}$, Eq. (6) \\
\hline $\mathrm{m}_{1}, \mathrm{~m}_{2}$ & $0.3,1$ & - & Increase in $\mathrm{q}_{\text {mid }}$ per unit $\mathrm{J}_{1}$ and $\mathrm{J}_{2}$, Eq. (7), $\mathrm{m}_{1,2}=\mathrm{a}_{1,2}-\mathrm{d}_{1,2}$ \\
\hline $\mathrm{r}_{0}$ & 1 & - & Background mean U/L ratio, Eq.(10) \\
\hline$\gamma_{0}$ & 0 & - & $\left(1-r_{0}\right) /\left(1+r_{0}\right)$ \\
\hline $\mathrm{r}_{\mathrm{q}}$ & 0.7 & $1 /$ day & Dependence of the U/L ratio on moisture deficit, Eq.(10) \\
\hline$\gamma_{\mathrm{q}}$ & 0.7 & 1/day & $2 \mathrm{r}_{\mathrm{q}} /\left(1+\mathrm{r}_{0}\right)$ \\
\hline $\mathrm{F}$ & 4 & - & $\begin{array}{l}\text { Ratio between saturation moist static energy and } \\
\text { temperature, Eq. (14) }\end{array}$ \\
\hline $\mathrm{f}$ & 0.5 & - & Relative weight used to linearly combine $\mathrm{T}_{1}$ and $\mathrm{T}_{2}$, Eq. (14) \\
\hline$\tau_{\mathrm{L}}$ & $1 / 12$ & day & Adjustment time to approach QE over the lower troposphere \\
\hline $\mathrm{c}_{1}, \mathrm{c}_{2}$ & $1,0.5$ & $50 \mathrm{~m} / \mathrm{s}$ & Dry speeds of the first and second mode \\
\hline$\varepsilon$ & $1 / 10$ & 1/day & Damping coefficient \\
\hline
\end{tabular}

